Multivariate normalized Powell-Sabin B-splines and quasi-interpolants

\author{
Hendrik Speleers
}

Report TW609, February 2012

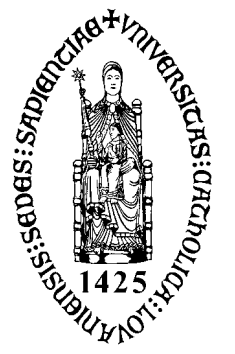

Katholieke Universiteit Leuven

Department of Computer Science

Celestijnenlaan 200A - B-3001 Heverlee (Belgium) 


\title{
Multivariate normalized Powell-Sabin B-splines and quasi-interpolants
}

\author{
Hendrik Speleers
}

Report TW 609, February 2012

Department of Computer Science, K.U.Leuven

\begin{abstract}
We present the construction of a multivariate normalized B-spline basis for the quadratic $C^{1}$-continuous spline space defined over a triangulation in $\mathbb{R}^{s}(s \geq 1)$ with a generalized Powell-Sabin refinement. The basis functions have a local support, they are nonnegative, and they form a partition of unity. The construction can be interpreted geometrically as the determination of a set of $s$-simplices that must contain a specific set of points. We also propose a family of quasi-interpolants based on this multivariate Powell-Sabin B-spline representation. Their spline coefficients only depend on a set of local function values. The multivariate quasi-interpolants reproduce quadratic polynomials and have an optimal approximation order.
\end{abstract}

Keywords : Multivariate Powell-Sabin splines, normalized quadratic B-splines, quasi-interpolation, spline approximation.

MSC : Primary : 65D07, Secondary : 41A15, 65D17 


\title{
Multivariate normalized Powell-Sabin B-splines and quasi-interpolants
}

\author{
Hendrik Speleers \\ Department of Computer Science, Katholieke Universiteit Leuven \\ Celestijnenlaan 200A, B-3001 Leuven, Belgium
}

\begin{abstract}
We present the construction of a multivariate normalized B-spline basis for the quadratic $C^{1}$-continuous spline space defined over a triangulation in $\mathbb{R}^{s}(s \geq 1)$ with a generalized Powell-Sabin refinement. The basis functions have a local support, they are nonnegative, and they form a partition of unity. The construction can be interpreted geometrically as the determination of a set of $s$-simplices that must contain a specific set of points. We also propose a family of quasi-interpolants based on this multivariate Powell-Sabin B-spline representation. Their spline coefficients only depend on a set of local function values. The multivariate quasiinterpolants reproduce quadratic polynomials and have an optimal approximation order.
\end{abstract}

Keywords: multivariate Powell-Sabin splines, normalized quadratic B-splines, quasi-interpolation, spline approximation

AMS classification: $65 \mathrm{D} 07,65 \mathrm{D} 17,41 \mathrm{~A} 15$

\section{Introduction}

Quasi-interpolation is a general term covering the construction of efficient local approximants to a given set of data or to a given function. The main interest of quasi-interpolants is that they are obtained without solving any global system of linear equations. Approximation methods using only local information are important in the context of real-time reconstruction and processing of large streams of (high-dimensional) data. Usually, a quasi-interpolant to a function $f$ is obtained as a linear combination of a set of basis functions forming a convex partition of unity and having a local support, in order to ensure stability and local control. The coefficients of such a linear combination depend on local values of $f$, and/or its derivatives, and/or its integrals (see, e.g., [17] and references quoted therein).

In this paper we address the problem of constructing multivariate quasi-interpolants in the space of quadratic splines defined over a generalized Powell-Sabin refinement of a triangulation in $\mathbb{R}^{s}$ $(s \geq 1)$. Originally, Powell and Sabin [12] developed a refinement for triangulations in $\mathbb{R}^{2}$, where every triangle in the triangulation is split into six subtriangles. Worsey and Piper [24] extended this result to the case $s=3$. Each tetrahedron is then refined into 24 subtetrahedra. This refinement has been generalized to $\mathbb{R}^{s}$ in [1] and thoroughly analyzed in [20]. Each simplex is partitioned into $(s+1)$ ! smaller simplices. In order to construct $C^{1}$-continuous quadratic splines over such a refined tessellation, certain geometric constraints on the positions of the split points must be satisfied. These geometric constraints can always be satisfied in the case $s=2$, but it remains an open question whether or not they can always be satisfied for an arbitrary tessellation in $\mathbb{R}^{s}$ with $s>2$. In $[20]$ it is shown that these constraints can be met for specific configurations important in practical applications. 
In the first part of the paper a normalized B-spline representation is developed for the multivariate $C^{1}$-continuous quadratic spline space defined over a triangulation in $\mathbb{R}^{s}$ with a generalized PowellSabin refinement. We present a method for constructing a set of locally supported basis functions that are nonnegative and form a partition of unity. Geometrically, the construction is equivalent to determining a set of small simplices that must contain a specific set of points. The method is a natural generalization of the approach given by Dierckx [6] for $s=2$ and by Sbibih et al. [19] for $s=3$. Similar B-spline representations for bivariate Powell-Sabin splines with higher smoothness have been considered in [21, 23], and for bivariate Clough-Tocher splines in [22].

In the second part of the paper we use this B-spline representation for constructing multivariate spline quasi-interpolants. The local Hermite interpolant described in [20] can be seen as a particular quasi-interpolant in the same spline space. Here, we propose a family of multivariate spline quasi-interpolants that only require function values (and not derivatives of the function $f$ ). They provide the full approximation order in the spline space. Bivariate quadratic Powell-Sabin quasi-interpolants have been developed in [10, 18], and trivariate Powell-Sabin quasi-interpolants in [19]. There exist many other quadratic quasi-interpolants, for example in two variables [3, 13] and in three variables $[11,14,15]$.

The paper is organized as follows. In Section 2 we briefly consider some preliminary concepts of multivariate polynomials in Bernstein-Bézier form. We also recall the definition of the quadratic spline space over a multi-dimensional Powell-Sabin refinement of a triangulation. Section 3 is devoted to the construction of a normalized basis for this spline space. We give a geometric interpretation in terms of simplices that must contain a specific set of points. We also show the relation between this normalized basis in one dimension and the classical univariate B-spline basis of degree two. In Section 4 we discuss a family of multivariate quasi-interpolants that reproduce quadratic polynomials. We also provide an upper bound for their infinity norm.

\section{Multivariate Powell-Sabin splines}

\subsection{Polynomials on simplices}

Let $\mathbf{V}_{0}, \ldots, \mathbf{V}_{s}$ be a set of linearly independent points in $\mathbb{R}^{s}, s>0$. An $s$-simplex $\mathcal{T}^{s}$ is defined as the convex hull of $s+1$ linearly independent points, i.e.,

$$
\mathcal{T}^{s}=\left[\mathbf{V}_{0}, \ldots, \mathbf{V}_{s}\right]=\left\{\sum_{i=0}^{s} \lambda_{i} \mathbf{V}_{i}: \sum_{i=0}^{s} \lambda_{i}=1, \lambda_{i} \geq 0\right\}
$$

The convex hull of any nonempty subset (of size $m+1$ ) of the $s+1$ points defining an $s$-simplex $\mathcal{T}^{s}$ is called an $m$-face of $\mathcal{T}^{s}$. In particular, 0 -faces are referred to as vertices, 1 -faces as edges, $(s-1)$-faces as facets of $\mathcal{T}^{s}$, and the $s$-face is $\mathcal{T}^{s}$ itself.

Barycentric coordinates provide an elegant tool for defining points inside a simplex. The barycentric coordinates $\tau=\left(\tau_{0}, \tau_{1}, \ldots, \tau_{s}\right)$ of a point $\mathbf{P}$ with respect to simplex $\mathcal{T}^{s}$ in $(2.1)$ are defined by

$$
\mathbf{P}=\sum_{i=0}^{s} \tau_{i} \mathbf{V}_{i}, \quad \text { and } \quad \sum_{i=0}^{s} \tau_{i}=1
$$

If $\mathbf{P}$ lies inside $\mathcal{T}^{s}$, then its barycentric coordinates are all positive.

Let $\mathbb{P}_{d}^{s}$ denote the linear space of $s$-variate polynomials of total degree less than or equal to $d$. Following the standard multi-index notation, we compactly write

$$
|\lambda|=\lambda_{0}+\ldots+\lambda_{s}, \quad \lambda !=\lambda_{0} ! \ldots \lambda_{s} !, \quad(\tau)^{\lambda}=\left(\tau_{0}\right)^{\lambda_{0}} \ldots\left(\tau_{s}\right)^{\lambda_{s}}
$$




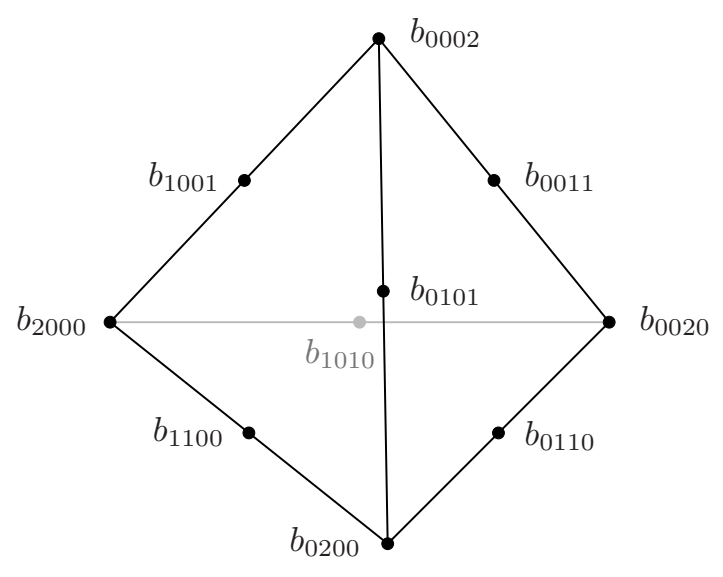

Figure 1: Schematic representation of the Bézier ordinates of a trivariate quadratic polynomial with respect to a tetrahedron.

for $\lambda=\left(\lambda_{0}, \lambda_{1}, \ldots, \lambda_{s}\right)$ and $\tau=\left(\tau_{0}, \tau_{1}, \ldots, \tau_{s}\right)$. Given a multi-index $\lambda$ with $|\lambda|=d$, the Bernstein basis polynomial $B_{\lambda}^{d}$ of degree $d$ with respect to the $s$-simplex $\mathcal{T}^{s}$ is defined as

$$
B_{\lambda}^{d}(\mathbf{P})=B_{\lambda}^{d}(\tau)=\frac{d !}{\lambda !}(\tau)^{\lambda} .
$$

Here, $\tau$ are the barycentric coordinates of the point $\mathbf{P}$ with respect to $\mathcal{T}^{s}$. The Bernstein basis polynomials form a partition of unity and they are nonnegative on their domain simplex $\mathcal{T}^{s}$. Because they form a basis of $\mathbb{P}_{d}^{s}$, each polynomial $p_{d} \in \mathbb{P}_{d}^{s}$ has a unique Bernstein-Bézier representation with respect to $\mathcal{T}^{s}$,

$$
p_{d}(\mathbf{P})=\sum_{|\lambda|=d} b_{\lambda} B_{\lambda}^{d}(\mathbf{P}) .
$$

The coefficients $b_{\lambda}$ are called Bézier ordinates. A schematic representation of these coefficients $b_{\lambda}$ is obtained by associating them to the so-called Bézier domain points $\xi_{\lambda}$ defined by

$$
\xi_{\lambda}=\frac{1}{|\lambda|} \sum_{i=0}^{s} \lambda_{i} \mathbf{V}_{i} .
$$

This representation is shown in Figure 1 for the case $s=3$ and $d=2$. We refer to [4] for more details on the Bernstein-Bézier representation of multivariate polynomials.

\subsection{The MPS-split}

Bangert and Prautzsch proposed in [1] a specific split of an $s$-simplex $\mathcal{T}^{s}$ into $(s+1)$ ! subsimplices. This split will be referred to as multi-dimensional Powell-Sabin (MPS) split since it is a natural generalization in $\mathbb{R}^{s}$ of the two-dimensional Powell-Sabin split [12]. The three-dimensional case has been described in [24].

Definition 2.1. The generic MPS-split of a simplex is recursively defined by increasing the dimension from one to the requested dimension: 
1. MPS-split of a line segment $\mathcal{T}^{1}$ (initialization, $s=1$ ):

(a) Select an interior point of $\mathcal{T}^{1}$.

(b) The split consists of the two obtained subsegments.

2. MPS-split of a simplex $\mathcal{T}^{s}$ (recursive case, $\left.s \geq 2\right)$ :

(a) Split each facet of $\mathcal{T}^{s}$ into s! subsimplices using an MPS-split of dimension $s-1$.

(b) Select an interior point of $\mathcal{T}^{s}$.

(c) Join this interior point to all points used in the MPS-splits of the facets to form the edges of the subsimplices.

The split point that is chosen in the interior of the $m$-face $\left[\mathbf{V}_{i_{0}}, \ldots, \mathbf{V}_{i_{m}}\right]$ of $\mathcal{T}^{s}$, with $1 \leq m \leq s$, will be denoted by $\mathbf{V}_{i_{0} \ldots i_{m}}$. The MPS-split is illustrated in Figure 2 for $s=1$ and $s=2$, and it is partially illustrated in Figure 3 for $s=3$. The latter figure only shows the edges of the twelve subtetrahedra built on the two visible faces of a given tetrahedron $\left[\mathbf{V}_{0}, \mathbf{V}_{1}, \mathbf{V}_{2}, \mathbf{V}_{3}\right]$.

Let $\mathcal{I}\left(\mathcal{T}^{s}\right)$ be the set of multi-indices describing all faces of $s$-simplex $\mathcal{T}^{s}$ :

$$
\mathcal{I}\left(\mathcal{T}^{s}\right)=\left\{\left(i_{0}, \ldots, i_{m}\right):\left[\mathbf{V}_{i_{0}}, \ldots, \mathbf{V}_{i_{m}}\right] \text { is an } m \text {-face of } \mathcal{T}^{s} \text { with } 0 \leq m \leq s\right\} .
$$

In order to specify explicitly the number of entries in a multi-index, we will use a notation with a superscript. For example, $i^{(m)}$ stands for a multi-index with $m+1$ entries. Given two multi-indices $i^{(m)}=\left(i_{0}, \ldots, i_{m}\right)$ and $j^{(n)}=\left(j_{0}, \ldots, j_{n}\right)$, we say that $i^{(m)} \subset j^{(n)}$ if $\left\{i_{0}, \ldots, i_{m}\right\} \subset\left\{j_{0}, \ldots, j_{n}\right\}$. In $[20]$ it is shown that the set of $(s+1)$ ! simplices

$$
\left\{\left[\mathbf{V}_{i^{(0)}}, \ldots, \mathbf{V}_{i^{(s)}}\right]: \text { for all } i^{(k)} \in \mathcal{I}\left(\mathcal{T}^{s}\right), k=0, \ldots, s, \text { and } i^{(k)} \subset i^{(k+1)}, k=0, \ldots, s-1\right\}
$$

forms the MPS-split of $\mathcal{T}^{s}$ and it is a valid $s$-dimensional triangulation.

So far, the construction of the MPS-split puts no constraints on the choices of the interior points used in the splitting. However, in order to construct a quadratic $C^{1}$-continuous spline over a triangulation with MPS-refinement, the following geometric constraints must be satisfied by the split points, see [20].

Definition 2.2. Given a triangulation $\Delta^{s}$ of a domain $\Omega$ in $\mathbb{R}^{s}$ consisting of $n_{t}$ simplices, the constrained MPS-refinement $\Delta^{s, *}$ of $\Delta^{s}$ into $n_{t}(s+1)$ ! subsimplices is constructed by applying Definition 2.1 on each simplex of $\Delta^{s}$ such that the following geometric constraints are satisfied:

1. For any simplex $\mathcal{T}^{s}$ in $\Delta^{s}$, each interior point chosen for an $m$-face $\mathcal{F}$ of $\mathcal{T}^{s}$ is $(s-m)$ coplanar with the interior points chosen for all $k$-faces, $k=m+1, \ldots, s$, containing $\mathcal{F}$ as a face.

2. When splitting any two neighbouring simplices in $\Delta^{s}$, the interior points chosen in both are collinear with the interior point chosen for the common facet.

These constraints are automatically satisfied for $s=1$. For $s=2$ they can be satisfied by choosing the incentres of the triangles as interior points in the splitting process [16]. It is still an open problem whether or not these constraints can always be met for an arbitrary triangulation in $\mathbb{R}^{s}$ with $s>2$. In [20] it is shown that this problem is feasible in the important special case where the vertices lie on a uniform regular lattice. This situation is of practical interest since in many applications (higher-dimensional) data are prescribed on a uniform grid. The first constraint in Definition 2.2 is illustrated in Figure 3 for two visible faces of a given tetrahedron. 

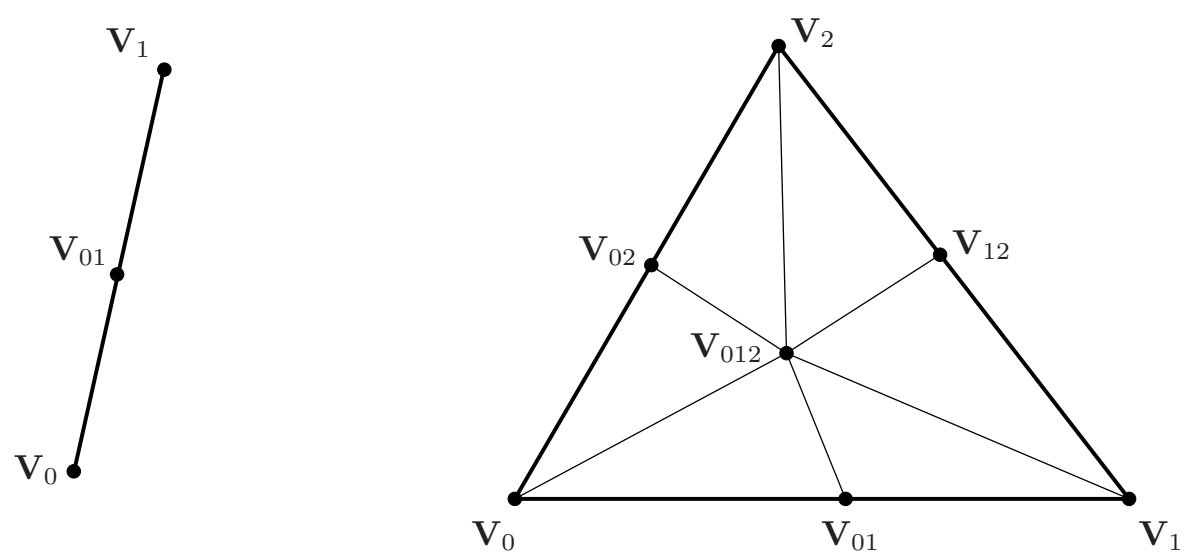

Figure 2: MPS-split of a line segment $(s=1$, left $)$ and a triangle $(s=2$, right $)$.

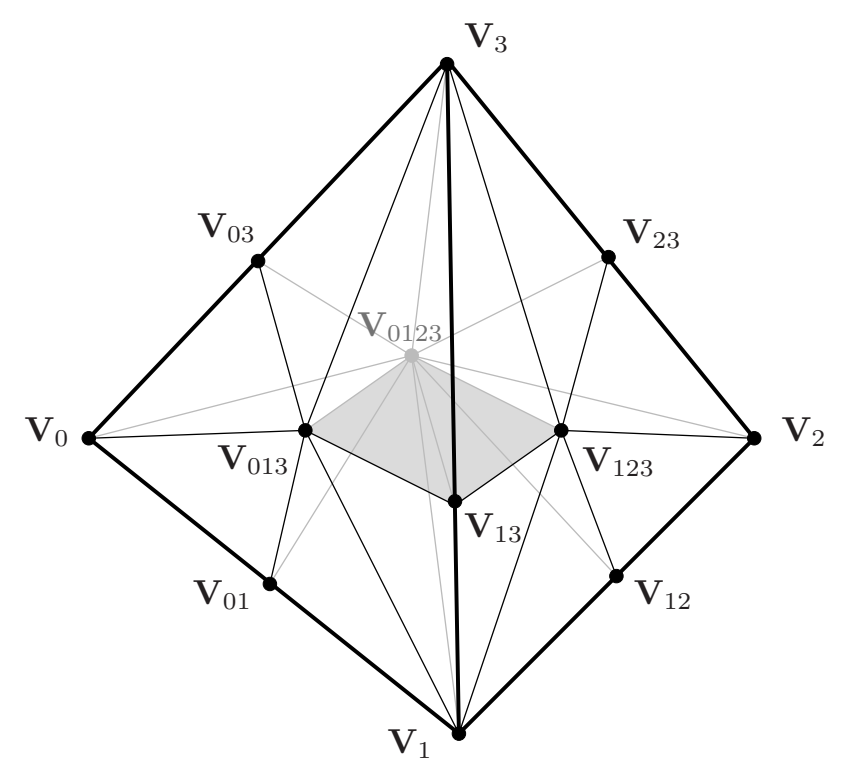

Figure 3: Three-dimensional PS-split, only shown for the two visible faces of the tetrahedron $\left[\mathbf{V}_{0}, \mathbf{V}_{1}, \mathbf{V}_{2}, \mathbf{V}_{3}\right]$. The interior split point $\mathbf{V}_{0123}$ is joined to all points on the boundary. The geometric constraint on the position of the split points is illustrated for the two visible faces: the point $\mathbf{V}_{13}$ on the common edge must be coplanar with the points $\mathbf{V}_{013}, \mathbf{V}_{123}$ and $\mathbf{V}_{0123}$ (shaded region).

\subsection{MPS-splines and their Bernstein-Bézier representation}

Let $\Delta^{s}$ be a triangulation consisting of $n_{t}$ simplices and $n_{v}$ vertices. Given a constrained MPSrefinement $\Delta^{s, *}$ of $\Delta^{s}$, see Definition 2.2, the multivariate Powell-Sabin spline is a piecewise quadratic $C^{1}$-continuous polynomial over $\Delta^{s, *}$. Such a spline $s_{\text {MPS }}$ is uniquely defined by means of the following Hermite interpolation problem [20]:

$$
s_{\mathrm{MPS}}\left(\mathbf{V}_{l}\right)=f_{l}, \quad \text { and } \quad \frac{\partial}{\partial x_{k}} s_{\mathrm{MPS}}\left(\mathbf{V}_{l}\right)=f_{x_{k}, l}, \quad k=1, \ldots, s,
$$


given function value $f_{l}$ and gradient values $f_{x_{k}, l}$ at each vertex $\mathbf{V}_{l}, l=1, \ldots, n_{v}$, in $\Delta^{s}$. The Cartesian coordinates of point $\mathbf{V}_{i}$ are denoted by $\left(V_{i}^{x_{1}}, \ldots, V_{i}^{x_{s}}\right)$.

We now show how to compute the Bernstein-Bézier representation of the MPS-spline satisfying (2.9). This spline is a quadratic polynomial on each of the subsimplices in $\Delta^{s, *}$. We will use an index-notation for Bézier ordinates and domain points that is different from the standard notation mentioned in Section 2.1, but it will be better suited for dealing with quadratics on triangulations. A Bézier domain point is located either at a vertex $\mathbf{V}_{i^{(m)}}$ of a subsimplex in $\Delta^{s, *}$, or at the midpoint of a line segment connecting two vertices $\mathbf{V}_{i^{(m)}}$ and $\mathbf{V}_{j^{(n)}}$ in $\Delta^{s, *}$. These domain points will be denoted by

$$
\xi_{i^{(m)}}=\mathbf{V}_{i^{(m)}}, \quad \text { and } \quad \xi_{i^{(m)}, j^{(n)}}=\left(\mathbf{V}_{i^{(m)}}+\mathbf{V}_{j^{(n)}}\right) / 2 .
$$

For simplicity, we also use the notation $\xi_{i^{(m)}, i^{(m)}}$ for $\xi_{i^{(m)}}$. The associated Bézier ordinates are denoted by $b_{i(m)}$ and $b_{i(m), j(n)}$, respectively, using the same subscript indices. The Bézier ordinates can be computed as follows:

1. For each vertex $\mathbf{V}_{i}$ in $\Delta^{s}$ :

$$
b_{i}=f_{i} .
$$

2. For each vertex $\mathbf{V}_{i}$ in $\Delta^{s}$ and for each $m$-face $\mathcal{F}_{i^{(m)}}$ in $\Delta^{s}$ with $1 \leq m \leq s$ containing vertex $\mathbf{V}_{i}$, i.e. $i \subset i^{(m)}$, and its interior split point $\mathbf{V}_{i^{(m)}}$ :

$$
b_{i, i(m)}=b_{i}+\frac{1}{2}\left(\sum_{k=1}^{s} f_{x_{k}, i}\left(V_{i(m)}^{x_{k}}-V_{i}^{x_{k}}\right)\right) .
$$

3. For each $m$-face $\mathcal{F}_{i^{(m)}}$ in $\Delta^{s}$ with $1 \leq m \leq s$ and $i^{(m)}=\left(i_{0}, \ldots, i_{m}\right)$, let $\alpha_{i(m)}=\left(\alpha_{i_{0}}, \ldots, \alpha_{i_{m}}\right)$ be the barycentric coordinates of split point $\mathbf{V}_{i^{(m)}}$ relative to $\mathcal{F}_{i(m)}$, i.e.,

$$
\mathbf{V}_{i(m)}=\sum_{k=0}^{m} \alpha_{i_{k}} \mathbf{V}_{i_{k}}
$$

Then, for any $j^{(n)} \supseteq i^{(m)}$,

$$
\xi_{i(m), j(n)}=\sum_{k=0}^{m} \alpha_{i_{k}} \xi_{i_{k}, j^{(n)}}
$$

and

$$
b_{i(m), j(n)}=\sum_{k=0}^{m} \alpha_{i_{k}} b_{i_{k}, j^{(n)}} .
$$

In this Bernstein-Bézier form, the MPS-splines can be easily evaluated, manipulated and displayed, see e.g. [4].

\section{Normalized multivariate Powell-Sabin B-splines}

\subsection{A B-spline representation for MPS-splines}

In this section we look for a suitable representation for MPS-splines,

$$
s_{\mathrm{MPS}}(\mathbf{x})=\sum_{i=1}^{n_{v}} \sum_{j=0}^{s} c_{i, j} B_{i, j}(\mathbf{x}),
$$


on a given triangulation $\Delta^{s}$ and MPS-refinement $\Delta^{s, *}$ of domain $\Omega$ in $\mathbb{R}^{s}$, such that the basis functions have a local support and satisfy the conditions

$$
B_{i, j}(\mathbf{x}) \geq 0, \quad \text { and } \quad \sum_{i=1}^{n_{v}} \sum_{j=0}^{s} B_{i, j}(\mathbf{x})=1,
$$

for all $\mathrm{x} \in \Omega$. A system with these properties is often called a blending system. To obtain locally supported basis functions, we associate with each vertex $\mathbf{V}_{i}$ in $\Delta^{s}(s+1)$ linearly independent sets of $(s+1)$ elements: $\left\{\gamma_{i, j, k}, k=0, \ldots, s\right\}, j=0, \ldots, s$ and $i=1, \ldots, n_{v}$.

Definition 3.1. Given a set $\left\{\gamma_{i, j, k}, k=0, \ldots, s\right\}$, the spline $B_{i, j}(\mathbf{x})$ is defined as the unique solution of interpolation problem (2.9) with all $f_{l}=0$ and $f_{x_{k}, l}=0$, except for $l=i$, where $f_{i}=\gamma_{i, j, 0}$ and $f_{x_{k}, i}=\gamma_{i, j, k}, k=1, \ldots, s$. Such a spline will be called a B-spline with respect to vertex $\mathbf{V}_{i}$.

It is easy to prove that the spline $B_{i, j}(\mathbf{x})$ only has its support on the region $\Omega_{i}$ covered by the set of simplices in $\Delta^{s}$ containing the vertex $\mathbf{V}_{i}$.

We now examine for which choices of $\left\{\gamma_{i, j, k}, k=0, \ldots, s\right\}, j=0, \ldots, s$ and $i=1, \ldots, n_{v}$, these basis functions form a convex partition of unity (3.2). From Definition 3.1 we know that only $(s+1)$ B-splines have nonzero function value and gradient at vertex $\mathbf{V}_{i}$. It directly follows that the necessary and sufficient conditions to form a partition of unity are given by

$$
\sum_{j=0}^{s} \gamma_{i, j, 0}=1, \quad \text { and } \quad \sum_{j=0}^{s} \gamma_{i, j, k}=0, \quad k=1, \ldots, s
$$

for each $i=1, \ldots, n_{v}$. To derive the conditions ensuring nonnegativity, we focus on the B-spline $B_{i, j}(\mathbf{x})$. It is sufficient to impose that all Bézier ordinates of the B-spline be nonnegative. Let $\tilde{b}_{i(m)}$ and $\tilde{b}_{i^{(m)}, j^{(n)}}$ be the ordinates of the B-spline corresponding to the domain points (2.10). We know from the definition of $B_{i, j}(\mathbf{x})$ that the Bézier ordinates $\tilde{b}_{l}=0$ and $\tilde{b}_{l, l^{(m)}}=0$, for all $l \neq i$ and all $l^{(m)} \supset l$, see (2.11)-(2.12). Because the Bézier ordinates (2.15) are convex combinations of other ordinates, it suffices to request that

$$
\tilde{b}_{i}=\gamma_{i, j, 0} \geq 0, \quad \text { and } \quad \tilde{b}_{i, i(m)}=\gamma_{i, j, 0}+\frac{1}{2} \sum_{k=1}^{s} \gamma_{i, j, k}\left(V_{i(m)}^{x_{k}}-V_{i}^{x_{k}}\right) \geq 0
$$

for all $i^{(m)} \supset i$ where $\mathcal{F}_{i^{(m)}}$ is an $m$-face in $\Delta^{s}$ with $1 \leq m \leq s$ containing vertex $\mathbf{V}_{i}$. These constraints are also necessary. From the Bernstein-Bézier theory and (2.13)-(2.15), we know that

$$
B_{i, j}\left(\mathbf{V}_{i}\right)=\tilde{b}_{i}, \quad \text { and } \quad B_{i, j}\left(\mathbf{V}_{i(m)}\right)=\alpha_{i} \tilde{b}_{i, i(m)}, i^{(m)} \supset i,
$$

for some $\alpha_{i}>0$. Thus, if one of the conditions (3.4) is violated, the corresponding B-spline $B_{i, j}(\mathbf{x})$ has a negative value at the points $\mathbf{V}_{i}$ or $\mathbf{V}_{i(m)}$.

\subsection{A geometric interpretation}

We now provide a geometric interpretation for the conditions (3.3) and (3.4) ensuring the convex partition of unity. To this end, we define $(s+1)$ points $\mathbf{Q}_{i, j}, j=0, \ldots, s$, for each vertex $\mathbf{V}_{i}$ such that

$$
\sum_{i=1}^{n_{v}} \sum_{j=0}^{s} \mathbf{Q}_{i, j} B_{i, j}(\mathbf{x})=\mathbf{x}
$$


Using interpolation problem (2.9) and the definition of the B-splines, the Cartesian coordinates $\left(Q_{i, j}^{x_{1}}, \ldots, Q_{i, j}^{x_{s}}\right)$ of $\mathbf{Q}_{i, j}, j=0, \ldots, s$, can be obtained as the solution of the $s$ systems

$$
\sum_{j=0}^{s} \gamma_{i, j, 0} Q_{i, j}^{x_{k}}=V_{i}^{x_{k}}, \quad \sum_{j=0}^{s} \gamma_{i, j, k} Q_{i, j}^{x_{k}}=1, \quad \sum_{j=0}^{s} \gamma_{i, j, l} Q_{i, j}^{x_{k}}=0, \quad 1 \leq l \neq k \leq s,
$$

for $k=1, \ldots, s$. We can compactly write (3.3) and (3.6) in the following matrix notation

$$
G Q=V
$$

with

$$
G=\left[\begin{array}{cccc}
\gamma_{i, 0,0} & \gamma_{i, 1,0} & \ldots & \gamma_{i, s, 0} \\
\gamma_{i, 0,1} & \gamma_{i, 1,1} & \ldots & \gamma_{i, s, 1} \\
\vdots & \vdots & & \vdots \\
\gamma_{i, 0, s} & \gamma_{i, 1, s} & \ldots & \gamma_{i, s, s}
\end{array}\right], \quad Q=\left[\begin{array}{cccc}
1 & Q_{i, 0}^{x_{1}} & \ldots & Q_{i, 0}^{x_{s}} \\
1 & Q_{i, 1}^{x_{1}} & \ldots & Q_{i, 1}^{x_{s}} \\
\vdots & \vdots & & \vdots \\
1 & Q_{i, s}^{x_{1}} & \ldots & Q_{i, s}^{x_{s}}
\end{array}\right], \quad V=\left[\begin{array}{cccc}
1 & V_{i}^{x_{1}} & \ldots & V_{i}^{x_{s}} \\
0 & 1 & & 0 \\
\vdots & & \ddots & \\
0 & 0 & & 1
\end{array}\right]
$$

The $s$-simplex $t_{i}=\left[\mathbf{Q}_{i, 0}, \ldots, \mathbf{Q}_{i, s}\right]$ will be referred to as the MPS-simplex with respect to vertex $\mathbf{V}_{i}$.

The constraints (3.4) were obtained by requiring nonnegativity of the Bézier ordinates corresponding to the domain points $\xi_{i}\left(=\mathbf{V}_{i}\right)$ and $\xi_{i, i(m)}$ for all $i^{(m)} \supset i$. This is equivalent to the following geometric condition.

Theorem 3.1. The B-splines $B_{i, j}(\mathbf{x}), j=0, \ldots, s$, are nonnegative if and only if the MPS-simplex $t_{i}=\left[\mathbf{Q}_{i, 0}, \ldots, \mathbf{Q}_{i, s}\right]$ contains the Bézier domain points $\xi_{i}$ and $\xi_{i, i(m)}$ for all $i^{(m)} \supset i$ where $\mathcal{F}_{i^{(m)}}$ is an $m$-face in $\Delta^{s}$ with $1 \leq m \leq s$ containing vertex $\mathbf{V}_{i}$.

Proof. For simplicity of notation, we just focus on B-splines $B_{1, j}(\mathbf{x}), j=0, \ldots, s$, and check their nonnegativity on a single simplex $\mathcal{T}^{s}=\left[\mathbf{V}_{1}, \ldots, \mathbf{V}_{s+1}\right]$. We follow a similar idea as the one used in [6] for bivariate Powell-Sabin B-splines. We compute the barycentric coordinates $\left(\sigma_{0}, \ldots, \sigma_{s}\right)$ of the stated Bézier domain points with respect to the MPS-simplex $t_{1}$ and impose them to be nonnegative. Let $\left(\tau_{0}, \ldots, \tau_{s}\right)$ be the barycentric coordinates of the same points with respect to the simplex $\mathcal{T}^{s}$. Then, by $(2.2)$, it holds that

$$
\left[\begin{array}{c}
\sigma_{0} \\
\sigma_{1} \\
\vdots \\
\sigma_{s}
\end{array}\right]=A\left[\begin{array}{c}
\tau_{0} \\
\tau_{1} \\
\vdots \\
\tau_{s}
\end{array}\right]
$$

with

$$
A=\left[\begin{array}{cccc}
1 & 1 & \ldots & 1 \\
Q_{1,0}^{x_{1}} & Q_{1,1}^{x_{1}} & \ldots & Q_{1, s}^{x_{1}} \\
\vdots & \vdots & & \vdots \\
Q_{1,0}^{x_{s}} & Q_{1,1}^{x_{s}} & \ldots & Q_{1, s}^{x_{s}}
\end{array}\right]^{-1}\left[\begin{array}{cccc}
1 & 1 & \ldots & 1 \\
V_{1}^{x_{1}} & V_{2}^{x_{1}} & \ldots & V_{s+1}^{x_{1}} \\
\vdots & \vdots & & \vdots \\
V_{1}^{x_{s}} & V_{2}^{x_{s}} & \ldots & V_{s+1}^{x_{s}}
\end{array}\right]
$$

Using (3.7) and

$$
\left[\begin{array}{cccc}
1 & 0 & \ldots & 0 \\
V_{1}^{x_{1}} & 1 & & 0 \\
\vdots & & \ddots & \\
V_{1}^{x_{s}} & 0 & & 1
\end{array}\right]^{-1}=\left[\begin{array}{cccc}
1 & 0 & \ldots & 0 \\
-V_{1}^{x_{1}} & 1 & & 0 \\
\vdots & & \ddots & \\
-V_{1}^{x_{s}} & 0 & & 1
\end{array}\right]
$$


we have

$$
\begin{aligned}
& A=\left[\begin{array}{cccc}
\gamma_{1,0,0} & \gamma_{1,0,1} & \ldots & \gamma_{1,0, s} \\
\gamma_{1,1,0} & \gamma_{1,1,1} & \ldots & \gamma_{1,1, s} \\
\vdots & \vdots & & \vdots \\
\gamma_{1, s, 0} & \gamma_{1, s, 1} & \ldots & \gamma_{1, s, s}
\end{array}\right]\left[\begin{array}{cccc}
1 & 1 & \ldots & 1 \\
0 & V_{2}^{x_{1}}-V_{1}^{x_{1}} & \ldots & V_{s+1}^{x_{1}}-V_{1}^{x_{1}} \\
\vdots & \vdots & & \vdots \\
0 & V_{2}^{x_{s}}-V_{1}^{x_{s}} & \ldots & V_{s+1}^{x_{s}}-V_{1}^{x_{s}}
\end{array}\right] \\
& =\left[\begin{array}{cccc}
\gamma_{1,0,0} & \gamma_{1,0,0}+\sum_{k=1}^{s} \gamma_{1,0, k}\left(V_{2}^{x_{k}}-V_{1}^{x_{k}}\right) & \ldots & \gamma_{1,0,0}+\sum_{k=1}^{s} \gamma_{1,0, k}\left(V_{s+1}^{x_{k}}-V_{1}^{x_{k}}\right) \\
\gamma_{1,1,0} & \gamma_{1,1,0}+\sum_{k=1}^{s} \gamma_{1,1, k}\left(V_{2}^{x_{k}}-V_{1}^{x_{k}}\right) & \ldots & \gamma_{1,1,0}+\sum_{k=1}^{s} \gamma_{1,1, k}\left(V_{s+1}^{x_{k}}-V_{1}^{x_{k}}\right) \\
\vdots & \vdots & & \vdots \\
\gamma_{1, s, 0} & \gamma_{1, s, 0}+\sum_{k=1}^{s} \gamma_{1, s, k}\left(V_{2}^{x_{k}}-V_{1}^{x_{k}}\right) & \ldots & \gamma_{1, s, 0}+\sum_{k=1}^{s} \gamma_{1, s, k}\left(V_{s+1}^{x_{k}}-V_{1}^{x_{k}}\right)
\end{array}\right] .
\end{aligned}
$$

Substituting the barycentric coordinates $(1,0, \ldots, 0)$ of domain point $\xi_{1}=\mathbf{V}_{1}$ related to $\mathcal{T}^{s}$ into (3.8), we get

$$
\sigma_{j}=\gamma_{1, j, 0},
$$

for $j=0, \ldots, s$. Let $\left(\alpha_{0}, \ldots, \alpha_{s}\right)$ be the barycentric coordinates of $\mathbf{V}_{i(m)}$ related to $\mathcal{T}^{s}$, then the barycentric coordinates of domain point $\xi_{1, i(m)}=\left(\mathbf{V}_{1}+\mathbf{V}_{i^{(m)}}\right) / 2$ are $\left(1+\alpha_{0}, \ldots, \alpha_{s}\right) / 2$. Substituting them into (3.8), results in

$$
\begin{aligned}
\sigma_{j} & =\gamma_{1, j, 0}+\frac{1}{2} \sum_{l=1}^{s} \alpha_{l} \sum_{k=1}^{s} \gamma_{1, j, k}\left(V_{l+1}^{x_{k}}-V_{1}^{x_{k}}\right) \\
& =\gamma_{1, j, 0}+\frac{1}{2} \sum_{k=1}^{s} \gamma_{1, j, k} \sum_{l=1}^{s} \alpha_{l}\left(V_{l+1}^{x_{k}}-V_{1}^{x_{k}}\right) \\
& =\gamma_{1, j, 0}+\frac{1}{2} \sum_{k=1}^{s} \gamma_{1, j, k}\left(V_{i(m)}^{x_{k}}-V_{1}^{x_{k}}\right),
\end{aligned}
$$

for $j=0, \ldots, s$. We see that (3.10)-(3.11) are the Bézier ordinates (2.11)-(2.12) for the different B-splines $B_{1, j}, j=0, \ldots, s$. Imposing that these coordinates be nonnegative is equivalent to the constraints (3.4), which are necessary and sufficient conditions for nonnegativity of $B_{1, j}$. Nonnegative barycentric coordinates correspond to points inside the domain triangle.

The construction of a normalized B-spline basis satisfying (3.2) can be interpreted as finding a set of simplices that must contain a number of specified points. These points will be referred to as MPS-points. It is clear that this geometric problem always has a solution. Once such MPSsimplex has been chosen, the corresponding B-splines are determined by relation (3.7): the sets $\left\{\gamma_{i, j, k}, k=0, \ldots, s\right\}, j=0, \ldots, s$, in Definition 3.1 are found by $G=V Q^{-1}$.

\subsection{Control points of an MPS-spline}

Referring to the spline representation (3.1) and the definition of the MPS-simplices (3.5) we define control points as

$$
\mathbf{c}_{i, j}=\left(Q_{i, j}^{x_{1}}, \ldots, Q_{i, j}^{x_{s}}, c_{i, j}\right),
$$

for $i=1, \ldots, n_{v}$ and $j=0, \ldots, s$. These control points are a natural multivariate generalization of the control points defined in [6] for the bivariate Powell-Sabin case. Since the normalized B-spline basis forms a convex partition of unity, it follows that the graph of spline (3.1) lies inside the convex hull of the control points (3.12). These points can be considered as vertices of the simplices $T_{i}=\left[\mathbf{c}_{i, 0}, \ldots, \mathbf{c}_{i, s}\right], i=1, \ldots, n_{v}$, which we will call control simplices. 

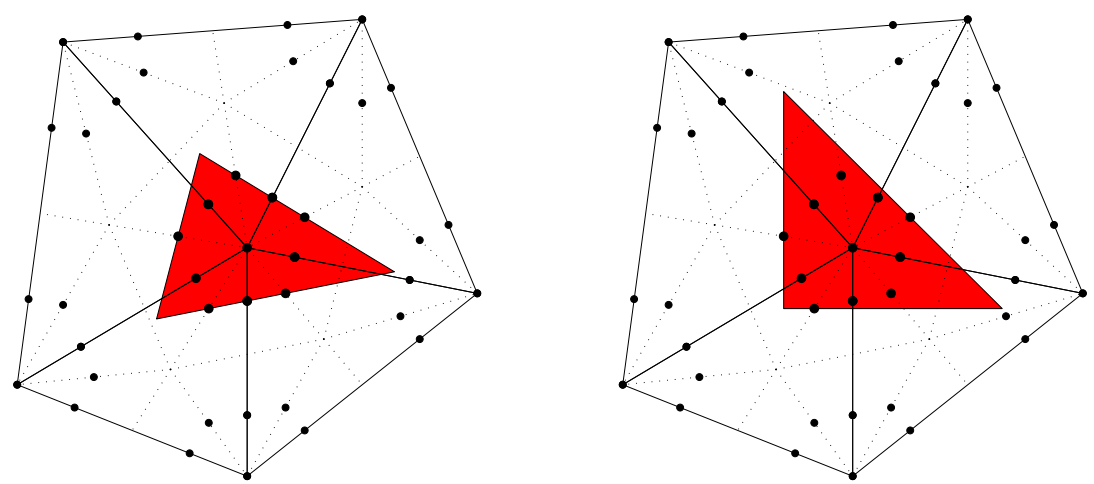

Figure 4: Location of two-dimensional MPS-points (black bullets), and two possible "valid" MPStriangles (shaded) associated with the central vertex in the triangulation. Left: MPS-triangle of minimal area. Right: right-angled isosceles MPS-triangle.

From the definition of the B-splines we know that

$$
s_{\mathrm{MPS}}\left(\mathbf{V}_{i}\right)=\sum_{j=0}^{s} \gamma_{i, j, 0} c_{i, j}, \quad \text { and } \quad \frac{\partial}{\partial x_{k}} s_{\mathrm{MPS}}\left(\mathbf{V}_{i}\right)=\sum_{j=0}^{s} \gamma_{i, j, k} c_{i, j}, \quad k=1, \ldots, s .
$$

By (3.7) we have $G^{-1}=Q V^{-1}$, and using (3.9) we get

$$
G^{-1}=\left[\begin{array}{cccc}
1 & Q_{i, 0}^{x_{1}}-V_{i}^{x_{1}} & \ldots & Q_{i, 0}^{x_{s}}-V_{i}^{x_{s}} \\
\vdots & \vdots & & \vdots \\
1 & Q_{i, s}^{x_{1}}-V_{i}^{x_{1}} & \ldots & Q_{i, s}^{x_{s}}-V_{i}^{x_{s}}
\end{array}\right] .
$$

Thus, by inverting system (3.13) and by (3.14), we obtain

$$
c_{i, j}=s_{\mathrm{MPS}}\left(\mathbf{V}_{i}\right)+\sum_{k=1}^{s}\left(Q_{i, j}^{x_{k}}-V_{i}^{x_{k}}\right) \frac{\partial}{\partial x_{k}} s_{\mathrm{MPS}}\left(\mathbf{V}_{i}\right),
$$

for $i=1, \ldots, n_{v}$ and $j=0, \ldots, s$. It directly follows that the control points $\mathbf{c}_{i, j}, j=0, \ldots, s$, belong to the hyper-plane in $\mathbb{R}^{s+1}$ tangent to the spline surface $s_{\text {MPS }}$ at vertex $\mathbf{V}_{i}$. Thus, control simplex $T_{i}$ is tangent to the spline surface at $\mathbf{V}_{i}$.

\subsection{Choice of MPS-simplices}

There are many MPS-simplices that satisfy the condition in Theorem 3.1. Figure 4 shows two examples for $s=2$. An appropriate choice, similar to the suggestion in [6] for PS-triangles in the bivariate case, is to calculate simplices with minimal hyper-volume. Small MPS-simplices ensure that the control points (3.12) will be close to the spline surface.

By (3.7), we have

$$
\left|\begin{array}{cccc}
1 & Q_{i, 0}^{x_{1}} & \ldots & Q_{i, 0}^{x_{s}} \\
1 & Q_{i, 1}^{x_{1}} & \ldots & Q_{i, 1}^{x_{s}} \\
\vdots & \vdots & & \vdots \\
1 & Q_{i, s}^{x_{1}} & \ldots & Q_{i, s}^{x_{s}}
\end{array}\right|=\left|\begin{array}{cccc}
\gamma_{i, 0,0} & \gamma_{i, 1,0} & \ldots & \gamma_{i, s, 0} \\
\gamma_{i, 0,1} & \gamma_{i, 1,1} & \ldots & \gamma_{i, s, 1} \\
\vdots & \vdots & & \vdots \\
\gamma_{i, 0, s} & \gamma_{i, 1, s} & \ldots & \gamma_{i, s, s}
\end{array}\right|^{-1}=\frac{1}{\operatorname{det}(G)},
$$


and it follows that the hyper-volume of the MPS-simplex $t_{i}$ is given by

$$
\operatorname{vol}\left(t_{i}\right)=\frac{1}{s !|\operatorname{det} G|} .
$$

Let $g(\gamma):=\operatorname{det}(G)$, which is a function with as arguments $\gamma_{i, 0,0}, \ldots, \gamma_{i, s, s}$. The construction of an MPS-simplex $t_{i}$ with minimal hyper-volume is equivalent to the following optimization problem:

$$
\arg \max _{\gamma} g(\gamma) \text {, subject to the linear constraints (3.3)-(3.4). }
$$

Note that we do not have to consider $|g(\gamma)|$ as objective function, because for each $\gamma$ there exists a $\tilde{\gamma}$ such that $g(\gamma)=-g(\tilde{\gamma})$. Figure 4 (left) illustrates an MPS-simplex of minimal hyper-volume for the case $s=2$.

This optimization problem can be quite complicated to solve, especially in high dimensions. Inspired by a similar idea given in $[6,19]$ for the bivariate and trivariate case, we now propose an alternative choice of MPS-simplex that only requires to solve a linear programming problem. Given $\varepsilon>0$, we assume

$$
\gamma_{i, j, j+1}=\varepsilon, \quad \text { and } \quad \gamma_{i, j, k}=0, \quad 1 \leq k \neq(j+1) \leq s,
$$

for $j=0, \ldots, s-1$. Because of (3.3), we have

$$
\gamma_{i, s, 0}=1-\sum_{j=0}^{s-1} \gamma_{i, j, 0}, \quad \text { and } \quad \gamma_{i, s, k}=-\varepsilon, \quad 1 \leq k \leq s .
$$

Note that the matrix $G$ in (3.7) simplifies by (3.19) to

$$
\begin{aligned}
G & =\left[\begin{array}{ccccc}
\gamma_{i, 0,0} & \gamma_{i, 1,0} & \ldots & \gamma_{i, s-1,0} & \gamma_{i, s, 0} \\
\varepsilon & 0 & \ldots & 0 & -\varepsilon \\
0 & \varepsilon & & 0 & -\varepsilon \\
\vdots & & \ddots & & \vdots \\
0 & 0 & & \varepsilon & -\varepsilon
\end{array}\right] \\
& =\left[\begin{array}{ccccc}
\gamma_{i, 0,0} & \gamma_{i, 1,0} & \ldots & \gamma_{i, s-1,0} & 1 \\
\varepsilon & 0 & \ldots & 0 & 0 \\
0 & \varepsilon & & 0 & 0 \\
\vdots & & \ddots & & \vdots \\
0 & 0 & & \varepsilon & 0
\end{array}\right]\left[\begin{array}{ccccc}
1 & 0 & \ldots & 0 & -1 \\
0 & 1 & \ldots & 0 & -1 \\
\vdots & & \ddots & & \vdots \\
0 & 0 & \ldots & 1 & -1 \\
0 & 0 & \ldots & 0 & 1
\end{array}\right],
\end{aligned}
$$

and its inverse

$$
G^{-1}=\left[\begin{array}{ccccc}
1 & 0 & \ldots & 0 & 1 \\
0 & 1 & \ldots & 0 & 1 \\
\vdots & & \ddots & & \vdots \\
0 & 0 & \ldots & 1 & 1 \\
0 & 0 & \ldots & 0 & 1
\end{array}\right]\left[\begin{array}{ccccc}
0 & 1 / \varepsilon & 0 & \ldots & 0 \\
0 & 0 & 1 / \varepsilon & & 0 \\
\vdots & \vdots & & \ddots & \\
0 & 0 & 0 & & 1 / \varepsilon \\
1 & -\gamma_{i, 0,0} / \varepsilon & -\gamma_{i, 1,0} / \varepsilon & \ldots & -\gamma_{i, s-1,0} / \varepsilon
\end{array}\right]
$$

Using relation (3.7), we get

$$
Q=G^{-1} V=\left[\begin{array}{ccccc}
1 & V_{i}^{x_{1}}+\left(1-\gamma_{i, 0,0}\right) / \varepsilon & V_{i}^{x_{2}}-\gamma_{i, 1,0} / \varepsilon & \cdots & V_{i}^{x_{s}}-\gamma_{i, s-1,0} / \varepsilon \\
1 & V_{i}^{x_{1}}-\gamma_{i, 0,0} / \varepsilon & V_{i}^{x_{2}}+\left(1-\gamma_{i, 1,0}\right) / \varepsilon & & V_{i}^{x_{s}}-\gamma_{i, s-1,0} / \varepsilon \\
\vdots & \vdots & & \ddots & \\
1 & V_{i}^{x_{1}}-\gamma_{i, 0,0} / \varepsilon & V_{i}^{x_{2}}-\gamma_{i, 1,0} / \varepsilon & & V_{i}^{x_{s}}+\left(1-\gamma_{i, s-1,0}\right) / \varepsilon \\
1 & V_{i}^{x_{1}}-\gamma_{i, 0,0} / \varepsilon & V_{i}^{x_{2}}-\gamma_{i, 1,0} / \varepsilon & \cdots & V_{i}^{x_{s}}-\gamma_{i, s-1,0} / \varepsilon
\end{array}\right] .
$$


We see that (3.19) leads to a right-angled MPS-simplex, with $\left\|\mathbf{Q}_{i, k}-\mathbf{Q}_{i, s}\right\|_{2}=1 / \varepsilon$ for each $k=0, \ldots, s-1$. In this case, we get

$$
g(\gamma)=\operatorname{det}(G)=(-\varepsilon)^{s} .
$$

Thus, the MPS-simplex of minimal hyper-volume satisfying (3.19) can be computed by finding the maximum of the linear function $\hat{g}(\gamma, \varepsilon)=\varepsilon$ under the linear constraints (3.3)-(3.4). Figure 4 (right) illustrates such a right-angled isosceles MPS-triangle for the case $s=2$.

As an obvious alternative, we could also take a negative value of $\varepsilon$ in (3.19), in which case we simply have to maximize the linear function $\bar{g}(\gamma, \varepsilon)=-\varepsilon$ under the linear constraints (3.3)-(3.4).

Finally, we remark that from a practical point of view it might be useful to select MPS-simplices of some special form. For example, in Section 4, we will suggest an alternative choice in the context of quasi-interpolation.

\subsection{Bézier ordinates of an MPS-spline in B-spline representation}

The Bézier ordinates of an MPS-spline in the representation (3.1)-(3.2) can be computed in a stable way from its B-spline coefficients $c_{i, j}$. Because of the local support of the B-splines, the Bézier ordinates $b_{i}$ and $b_{i, i(m)}$ in the neighbourhood of vertex $\mathbf{V}_{i}$, see (2.11)-(2.12), can be written as linear combinations of the $(s+1)$ spline coefficients $c_{i, k}, k=0, \ldots, s$. By (3.10)-(3.11) we know that the weights in these combinations can be interpreted as barycentric coordinates of the Bézier domain points $\xi_{i}$ and $\xi_{i, i(m)}$. We can summarize the computation of the Bézier ordinates given a spline representation (3.1)-(3.2) as follows:

1. For each vertex $\mathbf{V}_{i}$ in $\Delta^{s}$ :

$$
b_{i}=\sum_{j=0}^{s} \gamma_{i, j, 0} c_{i, j}
$$

with $\left(\gamma_{i, 0,0}, \ldots, \gamma_{i, s, 0}\right)$ the barycentric coordinates of $\mathbf{V}_{i}$ relative to MPS-simplex $t_{i}$.

2. For each vertex $\mathbf{V}_{i}$ in $\Delta^{s}$ and for each $m$-face $\mathcal{F}_{i^{(m)}}$ in $\Delta^{s}$ with $1 \leq m \leq s$ containing vertex $\mathbf{V}_{i}$, i.e. $i \subset i^{(m)}$ :

$$
b_{i, i(m)}=\sum_{j=0}^{s} \sigma_{i, i(m), j} c_{i, j},
$$

with $\left(\sigma_{i, i(m), 0}, \ldots, \sigma_{i, i^{(m)}, s}\right)$ the barycentric coordinates of Bézier domain point $\xi_{i, i(m)}$ relative to MPS-simplex $t_{i}$.

3. For each $m$-face $\mathcal{F}_{i^{(m)}}$ in $\Delta^{s}$ with $1 \leq m \leq s$ and $i^{(m)}=\left(i_{0}, \ldots, i_{m}\right)$, and for any $j^{(n)} \supseteq i^{(m)}$ :

$$
b_{i(m), j(n)}=\sum_{k=0}^{m} \alpha_{i_{k}} b_{i_{k}, j^{(n)}}
$$

with $\left(\alpha_{i_{0}}, \ldots, \alpha_{i_{m}}\right)$ the barycentric coordinates of split point $\mathbf{V}_{i^{(m)}}$ relative to $\mathcal{F}_{i^{(m)}}$, see $(2.13)$, and $b_{i_{k}, j(n)}$ ordinates obtained by (3.23).

The combinations (3.22)-(3.24) are all convex, because the points $\mathbf{V}_{i}\left(=\xi_{i}\right)$ and $\xi_{i, i(m)}$ are contained in the MPS-simplex $t_{i}$ by Theorem 3.1, and the split point $\mathbf{V}_{i^{(m)}}$ is also in the interior of $\mathcal{F}_{i(m)}$ by Definition 2.1 . 


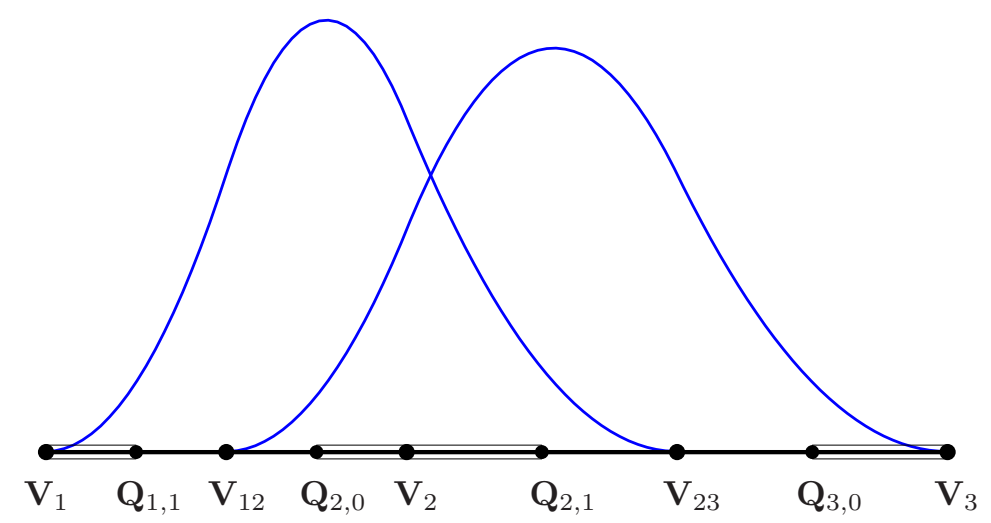

Figure 5: The two univariate basis functions $B_{2,0}$ and $B_{2,1}$, associated with vertex $\mathbf{V}_{2}$ and defined by means of the interval $\left[\mathbf{Q}_{2,0}, \mathbf{Q}_{2,1}\right]$ of minimal length. They are identical to the classical quadratic normalized B-splines defined over the knot sequence $\left\{\mathbf{V}_{1}, \mathbf{V}_{12}, \mathbf{V}_{2}, \mathbf{V}_{23}, \mathbf{V}_{3}\right\}$.

\subsection{The univariate case}

We now detail the construction of the normalized basis in one dimension $(s=1)$, and its relation with the classical B-spline basis [5]. Given a set of vertices $\mathbf{V}_{i} \in \mathbb{R}, i=1, \ldots, n_{v}$, and a set of split points $\mathbf{V}_{i-1, i} \in \mathbb{R}, i=2, \ldots, n_{v}$, constructed by Definition 2.1. In the one-dimensional case MPS-simplices are intervals, spanned by two points $\left[\mathbf{Q}_{i, 0}, \mathbf{Q}_{i, 1}\right]$ per vertex $\mathbf{V}_{i}$. Two basis functions $B_{i, j}, j=0,1$, are associated with each vertex $\mathbf{V}_{i}$. Their function and derivative value at $\mathbf{V}_{i}$ are denoted by $\gamma_{i, j, 0}$ and $\gamma_{i, j, 1}$, and they are related to the interval $\left[\mathbf{Q}_{i, 0}, \mathbf{Q}_{i, 1}\right]$ by

$$
\left[\begin{array}{cc}
\gamma_{i, 0,0} & \gamma_{i, 1,0} \\
\gamma_{i, 0,1} & \gamma_{i, 1,1}
\end{array}\right]=\left[\begin{array}{cc}
1 & \mathbf{V}_{i} \\
0 & 1
\end{array}\right]\left[\begin{array}{cc}
1 & \mathbf{Q}_{i, 0} \\
1 & \mathbf{Q}_{i, 1}
\end{array}\right]^{-1}=\frac{1}{\mathbf{Q}_{i, 1}-\mathbf{Q}_{i, 0}}\left[\begin{array}{cc}
\mathbf{Q}_{i, 1}-\mathbf{V}_{i} & \mathbf{V}_{i}-\mathbf{Q}_{i, 0} \\
-1 & 1
\end{array}\right]
$$

The MPS-intervals of minimal length are formed by the points

$$
\begin{array}{ll}
\mathbf{Q}_{1,0}=\mathbf{V}_{1}, & \mathbf{Q}_{1,1}=\left(\mathbf{V}_{1}+\mathbf{V}_{1,2}\right) / 2, \\
\mathbf{Q}_{i, 0}=\left(\mathbf{V}_{i-1, i}+\mathbf{V}_{i}\right) / 2, & \mathbf{Q}_{i, 1}=\left(\mathbf{V}_{i}+\mathbf{V}_{i, i+1}\right) / 2, \quad i=2, \ldots, n_{v}-1, \\
\mathbf{Q}_{n_{v}, 0}=\left(\mathbf{V}_{n_{v}-1, n_{v}}+\mathbf{V}_{n_{v}}\right) / 2, & \mathbf{Q}_{n_{v}, 1}=\mathbf{V}_{n_{v}} .
\end{array}
$$

The basis functions constructed by (3.25)-(3.26) are identical to the classical quadratic normalized B-splines, see Figure 5. For example, consider the basis function $B_{2,0}$ associated with vertex $\mathbf{V}_{2}$. Combining (2.11)-(2.15) with (3.25)-(3.26), its Bézier ordinates are given by

$$
\tilde{b}_{1}=\tilde{b}_{112}=0, \quad \tilde{b}_{12}=\frac{\mathbf{V}_{12}-\mathbf{V}_{1}}{\mathbf{V}_{2}-\mathbf{V}_{1}}, \quad \tilde{b}_{122}=1, \quad \tilde{b}_{2}=\frac{\mathbf{V}_{23}-\mathbf{V}_{2}}{\mathbf{V}_{23}-\mathbf{V}_{12}}, \quad \tilde{b}_{223}=\tilde{b}_{23}=\tilde{b}_{233}=\tilde{b}_{3}=0,
$$

corresponding to the Bézier domain points $\xi_{1}=\mathbf{V}_{1}, \xi_{112}=\left(\mathbf{V}_{1}+\mathbf{V}_{12}\right) / 2, \xi_{12}=\mathbf{V}_{12}, \xi_{122}=$ $\left(\mathbf{V}_{12}+\mathbf{V}_{2}\right) / 2, \xi_{2}=\mathbf{V}_{2}, \xi_{223}=\left(\mathbf{V}_{2}+\mathbf{V}_{23}\right) / 2, \xi_{23}=\mathbf{V}_{23}, \xi_{233}=\left(\mathbf{V}_{23}+\mathbf{V}_{3}\right) / 2$, and $\xi_{3}=\mathbf{V}_{3}$. Thus,

$$
B_{2,0}(\mathbf{x})= \begin{cases}\frac{\left(\mathbf{x}-\mathbf{V}_{1}\right)^{2}}{\left(\mathbf{V}_{2}-\mathbf{V}_{1}\right)\left(\mathbf{V}_{12}-\mathbf{V}_{1}\right)}, & \text { if } \mathbf{x} \in\left[\mathbf{V}_{1}, \mathbf{V}_{12}\right) \\ \frac{\left(\mathbf{x}-\mathbf{V}_{1}\right)\left(\mathbf{V}_{2}-\mathbf{x}\right)}{\left(\mathbf{V}_{2}-\mathbf{V}_{1}\right)\left(\mathbf{V}_{2}-\mathbf{V}_{12}\right)}+\frac{\left(\mathbf{V}_{23}-\mathbf{x}\right)\left(\mathbf{x}-\mathbf{V}_{12}\right)}{\left(\mathbf{V}_{23}-\mathbf{V}_{12}\right)\left(\mathbf{V}_{2}-\mathbf{V}_{12}\right)}, & \text { if } \mathbf{x} \in\left[\mathbf{V}_{12}, \mathbf{V}_{2}\right), \\ \frac{\left(\mathbf{V}_{23}-\mathbf{x}\right)^{2}}{\left(\mathbf{V}_{23}-\mathbf{V}_{12}\right)\left(\mathbf{V}_{23}-\mathbf{V}_{2}\right)}, & \text { if } \mathbf{x} \in\left[\mathbf{V}_{2}, \mathbf{V}_{23}\right), \\ 0, & \text { elsewhere, }\end{cases}
$$

which is the definition of a classical quadratic normalized B-spline defined over the knot sequence $\left\{\mathbf{V}_{1}, \mathbf{V}_{12}, \mathbf{V}_{2}, \mathbf{V}_{23}\right\}$, see [5]. Note that the points in (3.26) correspond to the classical Greville sites in the quadratic case. 


\section{Quasi-interpolation}

\subsection{Quasi-interpolants based on the B-spline representation}

In this section we describe how the normalized B-splines can be used to define quasi-interpolants, that are exact on the space of quadratic polynomials $\mathbb{P}_{2}^{s}$. In particular, we are interested in quasiinterpolants of the form

$$
\mathcal{Q} f=\sum_{i=1}^{n_{v}} \sum_{j=0}^{s} \mu_{i, j}(f) B_{i, j}
$$

where $\mu_{i, j}(f), i=1, \ldots, n_{v}$ and $j=0, \ldots, s$, are suitable linear functionals depending on function $f$.

Inspired by (2.9), the Hermite interpolant is a natural choice. Using (3.15), we have

$$
\mu_{i, j}^{(1)}(f)=f\left(\mathbf{V}_{i}\right)+\sum_{k=1}^{s}\left(Q_{i, j}^{x_{k}}-V_{i}^{x_{k}}\right) \frac{\partial}{\partial x_{k}} f\left(\mathbf{V}_{i}\right)
$$

This Hermite interpolation scheme reproduces $\mathbb{P}_{2}^{s}$, and it is shown in $[20]$ that it has an optimal approximation order.

The interpolant (4.2) requires the computation of $f$ and its derivatives at the vertices. We now describe how one can construct quasi-interpolants of the form (4.1) that reproduce $\mathbb{P}_{2}^{s}$ and do not need values of derivatives of $f$. We are looking for quasi-interpolants of the form (4.1) such that

$$
\mu_{i, j}^{(2)}(f)=\sum_{k=1}^{3} q_{i, j, k} f\left(\mathbf{Z}_{i, j, k}\right)
$$

given three points $\mathbf{Z}_{i, j, k} \in \mathbb{R}^{s}$ with Cartesian coordinates $\left(Z_{i, j, k}^{x_{1}}, \ldots, Z_{i, j, k}^{x_{s}}\right)$ and three values $q_{i, j, k} \in$ $\mathbb{R}, k=1,2,3$. To reproduce quadratic polynomials, the triple of points $\mathbf{Z}_{i, j, k} \in \mathbb{R}^{s}$ must satisfy a specific geometric configuration. This result was already observed in [10] for the bivariate case.

Theorem 4.1. Let $\mathcal{Q} f$ be a quasi-interpolant of the form (4.1) with (4.3). If

$$
\mathcal{Q} p=p, \quad \forall p \in \mathbb{P}_{2}^{s}
$$

then for any $1 \leq i \leq n_{v}, 0 \leq j \leq s$, and $\mathbf{Q}_{i, j} \neq \mathbf{V}_{i}$, the points

$$
\mathbf{V}_{i}, \quad \mathbf{Q}_{i, j}, \quad \text { and } \quad\left\{\mathbf{Z}_{i, j, k}, \text { for each } k \text { where } q_{i, j, k} \neq 0\right\}
$$

are collinear.

Proof. We must only treat the case $s>2$, because (4.5) is automatically satisfied for $s=1$, and it has been proved for $s=2$ in [10]. We know that (4.2) gives us the coefficients of the MPS-representation (3.1) of any polynomial in $\mathbb{P}_{2}^{s}$. It follows from (4.4) that

$$
\mu_{i, j}^{(2)}(p)=\mu_{i, j}^{(1)}(p), \quad \forall p \in \mathbb{P}_{2}^{s},
$$

with $i=1, \ldots, n_{v}$ and $j=0, \ldots, s$. We now fix $1 \leq i \leq n_{v}$ and $0 \leq j \leq s$, and we assume that $\mathbf{V}_{i}=(0, \ldots, 0)$ without loss of generality. Expanding (4.6) for the monomial basis 
$\left\{\left(x_{1}\right)^{a_{1}} \ldots\left(x_{s}\right)^{a_{s}}\right.$, for any $\left.0 \leq a_{1}+\ldots+a_{s} \leq 2\right\}$ results in the following system:

$$
\begin{aligned}
& \sum_{k=1}^{3} q_{i, j, k}=1, \\
& \sum_{k=1}^{3} q_{i, j, k} Z_{i, j, k}^{x_{l}}=Q_{i, j}^{x_{l}}, \quad l=1, \ldots, s, \\
& \sum_{k=1}^{3} q_{i, j, k} Z_{i, j, k}^{x_{l}} Z_{i, j, k}^{x_{m}}=0, \quad l=1, \ldots, s, \quad m=1, \ldots, s .
\end{aligned}
$$

Since $\mathbf{Q}_{i, j} \neq \mathbf{V}_{i}$, we know that

$$
\exists r \text { such that } Q_{i, j}^{x_{r}} \neq V_{i}^{x_{r}}=0 .
$$

Then, we select $(s-1)$ smaller systems, each consisting of six equations, according to a value $r$ that satisfies (4.8):

$$
\begin{aligned}
& \sum_{k=1}^{3} q_{i, j, k}=1, \\
& \sum_{k=1}^{3} q_{i, j, k} Z_{i, j, k}^{x_{l}}=Q_{i, j}^{x_{l}}, \quad \sum_{k=1}^{3} q_{i, j, k} Z_{i, j, k}^{x_{r}}=Q_{i, j}^{x_{r},} \\
& \sum_{k=1}^{3} q_{i, j, k}\left(Z_{i, j, k}^{x_{l}}\right)^{2}=0, \quad \sum_{k=1}^{3} q_{i, j, k} Z_{i, j, k}^{x_{l}} Z_{i, j, k}^{x_{r}}=0, \quad \sum_{k=1}^{3} q_{i, j, k}\left(Z_{i, j, k}^{x_{r}}\right)^{2}=0,
\end{aligned}
$$

for each $l=1, \ldots, s$ and $l \neq r$. It is obvious that any solution of the full system (4.7) must also be a solution of all the smaller systems (4.9). By the bivariate result proved in [10], we know that any smaller system (4.9) can have solutions only if the points

$$
(0,0), \quad\left(Q_{i, j}^{x_{l}}, Q_{i, j}^{x_{r}}\right), \quad \text { and } \quad\left\{\left(Z_{i, j, k}^{x_{l}}, Z_{i, j, k}^{x_{r}}\right), \text { for each } k \text { where } q_{i, j, k} \neq 0\right\}
$$

are collinear. From (4.8) it follows that there is a unique representation $Z_{i, j, k}^{x_{r}}=\zeta_{k} Q_{i, j}^{x_{r}}$ for some value of $\zeta_{k}$, and in combination with (4.10) we may conclude that for each $k$ where $q_{i, j, k} \neq 0$,

$$
Z_{i, j, k}^{x_{r}}=\zeta_{k} Q_{i, j}^{x_{r}}, \quad Z_{i, j, k}^{x_{l}}=\zeta_{k} Q_{i, j}^{x_{l}} .
$$

Since (4.11) must hold for any $l=1, \ldots, s, l \neq r$, we obtain that $\mathbf{Z}_{i, j, k}=\zeta_{k} \mathbf{Q}_{i, j}$, implying (4.5).

It is clear that if $\mathbf{Q}_{i, j}=\mathbf{V}_{i}$, then we must take $\mu_{i, j}^{(2)}(f)=f\left(\mathbf{V}_{i}\right)$ in (4.1). From Theorem 3.1 we know that this situation can only occur for boundary vertices. The following theorem states how $\mu_{i, j}^{(2)}(f)$ must be chosen when $\mathbf{Q}_{i, j}$ is different from $\mathbf{V}_{i}$.

Theorem 4.2. Let $\mathcal{Q} f$ be any quasi-interpolant of the form (4.1) with $\mu_{i, j}(f)$ defined as in (4.3), where for each $1 \leq i \leq n_{v}$ and $0 \leq j \leq s, \mathbf{Q}_{i, j} \neq \mathbf{V}_{i}$, and $\mathbf{Z}_{i, j, k}, k=1,2,3$, are three distinct points such that

given some $\zeta_{i, j, k} \in \mathbb{R}$, and

$$
\mathbf{Z}_{i, j, k}=\left(1-\zeta_{i, j, k}\right) \mathbf{V}_{i}+\zeta_{i, j, k} \mathbf{Q}_{i, j}
$$

$$
\begin{aligned}
q_{i, j, 1} & =\frac{\zeta_{i, j, 2}-\zeta_{i, j, 2} \zeta_{i, j, 3}+\zeta_{i, j, 3}}{\left(\zeta_{i, j, 1}-\zeta_{i, j, 2}\right)\left(\zeta_{i, j, 3}-\zeta_{i, j, 1}\right)}, \\
q_{i, j, 2}= & \frac{\zeta_{i, j, 1}-\zeta_{i, j, 1} \zeta_{i, j, 3}+\zeta_{i, j, 3}}{\left(\zeta_{i, j, 1}-\zeta_{i, j, 2}\right)\left(\zeta_{i, j, 2}-\zeta_{i, j, 3}\right)}, \\
q_{i, j, 3}= & \frac{\zeta_{i, j, 1}-\zeta_{i, j, 1} \zeta_{i, j, 2}+\zeta_{i, j, 2}}{\left(\zeta_{i, j, 2}-\zeta_{i, j, 3}\right)\left(\zeta_{i, j, 3}-\zeta_{i, j, 1}\right)},
\end{aligned}
$$


then

$$
\mathcal{Q} p=p, \quad \forall p \in \mathbb{P}_{2}^{s}
$$

Proof. Fix $1 \leq i \leq n_{v}$ and $0 \leq j \leq s$, and consider the triple of points $\mathbf{Z}_{i, j, k}, k=1,2,3$, given in (4.12). We assume that $\mathbf{V}_{i}=(0, \ldots, 0)$ without loss of generality. Imposing for each monomial $p_{\text {mon }}$ in $\mathbb{P}_{2}^{s}$ that

$$
\mu_{i, j}^{(2)}\left(p_{\text {mon }}\right)=\mu_{i, j}^{(1)}\left(p_{\text {mon }}\right),
$$

results in the system (4.7). By the choice (4.12) and the assumption $\mathbf{Q}_{i, j} \neq \mathbf{V}_{i}$, this system simplifies to

$$
\sum_{k=1}^{3} q_{i, j, k}=1, \quad \sum_{k=1}^{3} \zeta_{i, j, k} q_{i, j, k}=1, \quad \sum_{k=1}^{3}\left(\zeta_{i, j, k}\right)^{2} q_{i, j, k}=0,
$$

because $\mathbf{Q}_{i, j} \neq \mathbf{V}_{i}$. It is easy to verify that the values (4.13) are a solution of (4.14). Hence, the corresponding $\mu_{i, j}^{(2)}(p), i=1, \ldots, n_{v}$ and $j=0, \ldots, s$, give exactly the coefficients of any $p \in \mathbb{P}_{2}^{s}$ with respect to the MPS-representation (3.1).

The quasi-interpolants given in Theorem 4.2 are multivariate generalizations of the discrete quasiinterpolants developed in $[18,19]$. We now consider two particular choices of the points $\mathbf{Z}_{i, j, k}$, which were also described in [10] for the bivariate case.

- If we set $\zeta_{i, j, 1}=0\left(\mathbf{Z}_{i, j, 1}=\mathbf{V}_{i}\right), \zeta_{i, j, 2}=1\left(\mathbf{Z}_{i, j, 2}=\mathbf{Q}_{i, j}\right), \zeta_{i, j, 3} \neq\{0,1\}$ in Theorem 4.2, then

$$
q_{i, j, 1}=\frac{-1}{\zeta_{i, j, 3}}, \quad q_{i, j, 2}=\frac{-\zeta_{i, j, 3}}{1-\zeta_{i, j, 3}}, \quad q_{i, j, 3}=\frac{1}{\zeta_{i, j, 3}\left(1-\zeta_{i, j, 3}\right)} .
$$

For $\zeta_{i, j, 3}=-1$, we get $q_{i, j, 1}=1, q_{i, j, 2}=1 / 2, q_{i, j, 3}=-1 / 2$.

- If we set $\zeta_{i, j, 1}=\frac{-\zeta_{i, j, 2}}{1-\zeta_{i, j, 2}}, \zeta_{i, j, 2} \neq\{0,1,2\}$ in Theorem 4.2 , then

$$
q_{i, j, 1}=\frac{-\left(1-\zeta_{i, j, 2}\right)^{2}}{\zeta_{i, j, 2}\left(2-\zeta_{i, j, 2}\right)}, \quad q_{i, j, 2}=\frac{1}{\zeta_{i, j, 2}\left(2-\zeta_{i, j, 2}\right)}, \quad q_{i, j, 3}=0 .
$$

For this choice, the coefficient $\mu_{i, j}^{(2)}(f)$ in (4.3) only needs two evaluations of the function $f$, i.e. at the points $\mathbf{Z}_{i, j, 1}$ and $\mathbf{Z}_{i, j, 2}$. For $\zeta_{i, j, 1}=1 / 2$ and $\zeta_{i, j, 2}=-1$, we get $q_{i, j, 1}=4 / 3$, $q_{i, j, 2}=-1 / 3, q_{i, j, 3}=0$.

For some applications it may be too restrictive to request function evaluations at arbitrarily chosen points. However, it seems reasonable to assume that the function $f$ can be evaluated at vertices of $\Delta^{s}$ and at some (given) points on the edges of $\Delta^{s}$. The construction in Theorem 4.2 requires to evaluate $f$ at points collinear with the vertices of $\Delta^{s}$ and with the points $\mathbf{Q}_{i, j}$ defining the set of B-splines. So, it may be useful to select $\mathbf{Q}_{i, j}$ such that

$$
\mathbf{Q}_{i, j}=\left(1-\beta_{i, j}\right) \mathbf{V}_{i}+\beta_{i, j} \mathbf{V}_{l_{i, j}}
$$

for some vertex $\mathbf{V}_{l_{i, j}}$ of $\Delta^{s}$, see Figure 6 for the case $s=2$. The value $\beta_{i, j}$ is preferably selected such that the hyper-volume of the MPS-simplex $t_{i}$ is small given the constraints in Theorem 3.1. Then, we can choose the points $\mathbf{Z}_{i, j, k}$ in Theorem 4.2 as

$$
\mathbf{Z}_{i, j, 1}=\mathbf{V}_{i}, \quad \mathbf{Z}_{i, j, 2}=\mathbf{V}_{l_{i, j}}, \quad \mathbf{Z}_{i, j, 3}=\left(1-\rho_{i, j}\right) \mathbf{V}_{i}+\rho_{i, j} \mathbf{V}_{l_{i, j}},
$$

selecting $\rho_{i, j}$ such that $\mathbf{Z}_{i, j, 3}$ is a point where the value of $f$ is available.

Finally, we remark that due to the local nature of our family of quasi-interpolants described in Theorem 4.2, different choices of the coefficients $\mu_{i, j}(f)$ can be used for different vertices. 

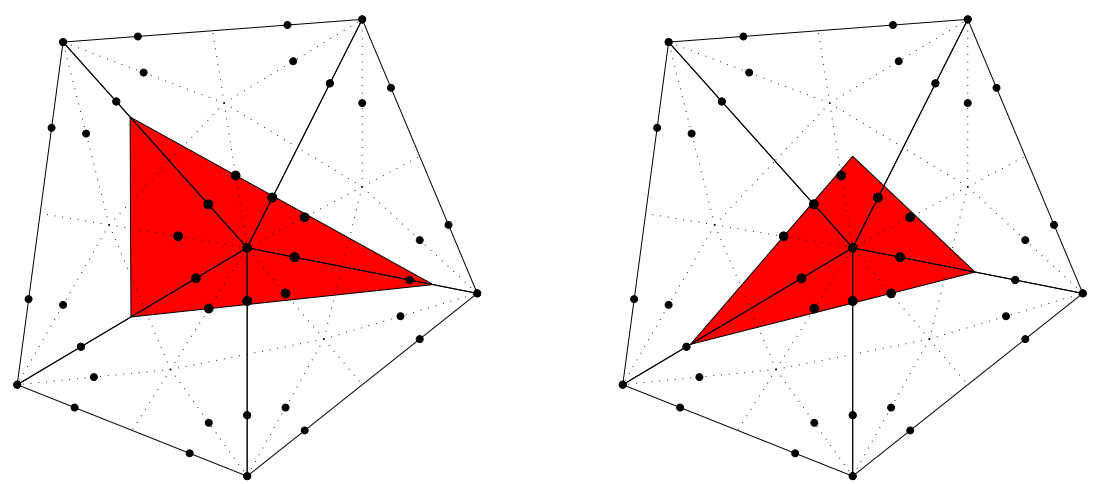

Figure 6: Two MPS-triangles (shaded) associated with the central vertex $\mathbf{V}_{i}$ in the given triangulation $\Delta$, such that the points $\mathbf{Q}_{i, j}$ belong to edges of $\Delta$ emanating from $\mathbf{V}_{i}$ or to their prolongation.

\subsection{Upper bound for the norm of the quasi-interpolants}

We now look for an upper bound for the infinity-norm of the quasi-interpolants constructed in Theorem 4.2, because such a bound can be used to investigate the approximation order of the quasi-interpolants. We assume that all points $\mathbf{Z}_{i, j, k}$ related to a vertex $\mathbf{V}_{i}$ are chosen in the region $\Omega_{i}$ covered by the union of simplices in $\Delta^{s}$ containing $\mathbf{V}_{i}$.

We know that a quasi-interpolant $\mathcal{Q}$ constructed as in Theorem 4.2 reproduces quadratic polynomials $p \in \mathbb{P}_{2}^{s}$. Let

$$
\|f\|_{\infty, \Upsilon}:=\sup _{\mathbf{x} \in \Upsilon}|f(\mathbf{x})|, \quad|f|_{m, \infty, \Upsilon}:=\max _{|\mathbf{a}|=m}\left\|\frac{\partial^{|\mathbf{a}|}}{\partial \mathbf{x}^{\mathbf{a}}} f\right\|_{\infty, \Upsilon},
$$

and

$$
\|\mathcal{Q}\|:=\max _{i=1, \ldots, n_{v}} \max _{j=0, \ldots, s} \sum_{k=1}^{3}\left|q_{i, j, k}\right| .
$$

From the convex partition of unity property of the normalized B-spline basis, see (3.2), it follows that the approximation error of any quasi-interpolant $\mathcal{Q}$ of the form (4.1), reproducing quadratic polynomials, with its coefficients given by (4.3) and with each $\mathbf{Z}_{i, j, k} \in \Omega_{i}$, can be bounded on any simplex $\mathcal{T}^{s} \in \Delta^{s}$ by

$$
\|\mathcal{Q} f-f\|_{\infty, \mathcal{T}^{s}} \leq\|\mathcal{Q}(f-p)\|_{\infty, \mathcal{T}^{s}}+\|p-f\|_{\infty, \mathcal{T}^{s}}, \quad p \in \mathbb{P}_{2}^{s},
$$

and

$$
\|\mathcal{Q} f-f\|_{\infty, \mathcal{T}^{s}} \leq(1+\|\mathcal{Q}\|) \inf _{p \in \mathbb{P}_{2}^{s}}\|f-p\|_{\infty, \Omega_{\mathcal{T}^{s}}},
$$

where $\Omega_{\mathcal{T}^{s}}$ is the region covered by the set of simplices in $\Delta^{s}$ having an $m$-face, $0 \leq m \leq s$, in common with $\mathcal{T}^{s}$. Using classical approximation theory, see $[2,7]$, we then obtain that

$$
\|\mathcal{Q} f-f\|_{\infty, \mathcal{T}^{s}} \leq C\left(d_{\Omega_{\mathcal{T}^{s}}}\right)^{3}(1+\|\mathcal{Q}\|)|f|_{3, \infty, \Omega_{\mathcal{T}^{s}}},
$$

with $d_{\Omega_{\mathcal{T}} s}$ the diameter of $\Omega_{\mathcal{T}^{s}}$. Here, $C$ depends on the so-called chunkiness $\gamma_{\Omega_{\mathcal{T}^{s}}}$ of $\Omega_{\mathcal{T}^{s}}$. Given a bounded domain $\Upsilon$ with diameter $d \Upsilon$, let $\rho_{\max , \Upsilon}$ be defined as the supremum of the diameters 
of all balls $\mathcal{B}$ such that for any $\mathbf{x} \in \Upsilon$, the convex hull of $\{\mathbf{x}\} \cup \mathcal{B}$ is a subset of $\Upsilon$. Then, the ratio $\gamma_{\Upsilon}=d_{\Upsilon} / \rho_{\max , \Upsilon}$ is referred to as the chunkiness of $\Upsilon$. We may conclude from (4.21) that such a quasi-interpolant $\mathcal{Q}$ with a bounded norm $\|\mathcal{Q}\|$ has an optimal approximation order of three. The following theorem provides an upper bound for $\|\mathcal{Q}\|$.

Theorem 4.3. Let $\mathcal{Q} f$ be any quasi-interpolant of the form (4.1), where the coefficients $\mu_{i, j}(f)$ are chosen as in (4.3) with (4.12)-(4.13). Let $K_{1} \geq 0$ and $K_{2}>0$ be constants such that

1. for each $\mathbf{Z}_{i, j, k}$ :

$$
\left\|\mathbf{Z}_{i, j, k}-\mathbf{V}_{i}\right\|_{2} \leq K_{1}\left\|\mathbf{Q}_{i, j}-\mathbf{V}_{i}\right\|_{2}
$$

2. for each couple of points $\mathbf{Z}_{i, j, k}$ and $\mathbf{Z}_{i, j, l}$ with $k \neq l$ :

$$
\left\|\mathbf{Z}_{i, j, k}-\mathbf{Z}_{i, j, l}\right\|_{2} \geq K_{2}\left\|\mathbf{Q}_{i, j}-\mathbf{V}_{i}\right\|_{2} .
$$

Then,

$$
\|\mathcal{Q}\| \leq K:=\frac{3 K_{1}\left(2+K_{1}\right)}{\left(K_{2}\right)^{2}}
$$

Proof. Fix $1 \leq i \leq n_{v}$ and $0 \leq j \leq s$. From (4.12) and the conditions (4.22)-(4.23), it follows

$$
\left|\zeta_{i, j, k}\right|=\frac{\left\|\mathbf{Z}_{i, j, k}-\mathbf{V}_{i}\right\|_{2}}{\left\|\mathbf{Q}_{i, j}-\mathbf{V}_{i}\right\|_{2}} \leq K_{1}
$$

for any $k=1,2,3$, and

$$
\left|\zeta_{i, j, k}-\zeta_{i, j, l}\right|=\frac{\left\|\mathbf{Z}_{i, j, k}-\mathbf{Z}_{i, j, l}\right\|_{2}}{\left\|\mathbf{Q}_{i, j}-\mathbf{V}_{i}\right\|_{2}} \geq K_{2}
$$

for any $k \neq l \in\{1,2,3\}$. Combining (4.13) and the bounds (4.25)-(4.26), we get

$$
\left|q_{i, j, k}\right| \leq \frac{2 K_{1}+\left(K_{1}\right)^{2}}{\left(K_{2}\right)^{2}},
$$

and the result (4.24) follows from (4.20).

As already noted in [10], once the family of B-splines has been selected (and so the points $\mathbf{Q}_{i, j}$ ), then the upper bound for the norm of $\mathcal{Q}$ in (4.24) decreases when the triple of points $\mathbf{Z}_{i, j, k}$, $k=1,2,3$, is chosen less localized. For example, if we take $\zeta_{i, j, 1}=0, \zeta_{i, j, 2}=\alpha$ and $\zeta_{i, j, 3}=-\alpha$, for all $i=1, \ldots, n_{v}$ and $j=0, \ldots, s$, then $K_{1}=K_{2}=\alpha$, and the upper bound in (4.24) becomes $K=3(2+\alpha) / \alpha$, which decreases when $\alpha$ increases.

On the other hand, if the points $\mathbf{Z}_{i, j, k}$ are fixed, then the upper bound for the norm of $\mathcal{Q}$ in (4.24) decreases as the distance between the points $\mathbf{Q}_{i, j}$ and vertex $\mathbf{V}_{i}$ decreases. For example, if this distance can be reduced by a factor $\frac{1}{\alpha}<1$, then we can multiply $K_{1}$ and $K_{2}$ by the factor $\alpha>1$, and the upper bound in (4.24) decreases to $K=\frac{3 K_{1}\left(2+\alpha K_{1}\right)}{\alpha\left(K_{2}\right)^{2}}$. Thus, the upper bound for the infinity-norm of the quasi-interpolation operator indicates that families of B-splines, defined by MPS-simplices $t_{i}$ with a small (or minimal) hyper-volume, are a good choice.

\subsection{Choice of MPS-simplices for quasi-interpolation}

We now propose a practical construction of the MPS-simplices satisfying (4.17). In order to ensure the nonnegativity of the basis functions $B_{i, j}, j=0, \ldots, s$, the MPS-simplex $t_{i}=\left[\mathbf{Q}_{i, 0}, \ldots, \mathbf{Q}_{i, s}\right]$ has to contain the Bézier domain points in the direct neighbourhood of vertex $\mathbf{V}_{i}$, see Theorem 3.1. In order to satisfy (4.17)-(4.18), we assume that there are at least $s+1$ edges with different directions 
in $\Delta^{s}$ emanating from any vertex $\mathbf{V}_{i}$. In Section 4.2 , we have also seen that the upper bound (4.24) for the norm of the quasi-interpolant benefits from a small distance between vertex $\mathbf{V}_{i}$ and any point $\mathbf{Q}_{i, j}$. For the sake of brevity, we only consider interior vertices $\mathbf{V}_{i}$ of $\Delta^{s}$, but the approach can be suitably adapted for boundary vertices.

We first derive an upper bound for $\left\|\mathbf{Q}_{i, j}-\mathbf{V}_{i}\right\|_{2}$. Let

$$
H_{i}=\max _{\mathbf{V}_{j} \in \Omega_{i}}\left\|\mathbf{V}_{j}-\mathbf{V}_{i}\right\|_{2}
$$

then it is easy to see that the $s$-dimensional ball centered in $\mathbf{V}_{i}$ with radius $H_{i} / 2$ contains all Bézier domain points which are direct neighbours of $\mathbf{V}_{i}$. Thus, the B-splines $B_{i, j}, j=0, \ldots, s$, will be nonnegative when the MPS-simplex $t_{i}$ contains this ball. Given a set of points $\mathbf{Q}_{i, j}, j=0, \ldots, s$, we define the following set of $s$-simplices:

$$
t_{i, j}^{Q}=\left[\mathbf{V}_{i}, \mathbf{Q}_{i, 0}, \ldots, \mathbf{Q}_{i, j-1}, \mathbf{Q}_{i, j+1}, \ldots, \mathbf{Q}_{i, s}\right],
$$

for $j=0, \ldots, s$. Let $h_{i, j}$ be the perpendicular distance from the vertex $\mathbf{V}_{i}$ to the $(s-1)$-face

$$
f_{i, j}^{Q}=\left[\mathbf{Q}_{i, 0}, \ldots, \mathbf{Q}_{i, j-1}, \mathbf{Q}_{i, j+1}, \ldots, \mathbf{Q}_{i, s}\right]
$$

of $t_{i, j}^{Q}$. This distance is related to the ratio of the hyper-volumes of $t_{i, j}^{Q}$ and $f_{i, j}^{Q}$ as follows (see, e.g., $[9])$ :

$$
h_{i, j}=s \frac{\operatorname{vol}\left(t_{i, j}^{Q}\right)}{\operatorname{vol}\left(f_{i, j}^{Q}\right)} .
$$

We know that the distance from $\mathbf{V}_{i}$ to any point of the facet $f_{i, j}^{Q}$ is larger than $h_{i, j}$. Thus, if vertex $\mathbf{V}_{i}$ is contained in simplex $\left[\mathbf{Q}_{i, 0}, \ldots, \mathbf{Q}_{i, s}\right]$ and

$$
h_{i, j} \geq \frac{H_{i}}{2}, \quad j=0, \ldots, s,
$$

then the simplex will satisfy the condition in Theorem 3.1.

We now consider a specific choice of the points $\mathbf{Q}_{i, j}$. Suppose

$$
\mathbf{Q}_{i, j}=\mathbf{V}_{i}+\delta_{i, j} \beta_{i} \mathbf{W}_{l_{i, j}}, \quad j=0, \ldots, s,
$$

for given $\beta_{i}>0, \delta_{i, j} \in\{-1,1\}$, and

$$
\mathbf{W}_{l_{i, j}}=\frac{\mathbf{V}_{l_{i, j}}-\mathbf{V}_{i}}{\left\|\mathbf{V}_{l_{i, j}}-\mathbf{V}_{i}\right\|_{2}},
$$

where $\mathbf{V}_{l_{i, j}}$ are vertices at the boundary of $\Omega_{i}$, such that the point $\mathbf{O}=(0, \ldots, 0)$ is contained in the $s$-simplex $\left[\delta_{i, 0} \mathbf{W}_{l_{i, 0}}, \ldots, \delta_{i, s} \mathbf{W}_{l_{i, s}}\right]$. Let $t_{i, j}$ and $f_{i, j}$ be the following simplices composed of the points given in (4.34):

$$
t_{i, j}=\left[\mathbf{O}, \delta_{i, 0} \mathbf{W}_{l_{i, 0}}, \ldots, \delta_{i, j-1} \mathbf{W}_{l_{i, j-1}}, \delta_{i, j+1} \mathbf{W}_{l_{i, j+1}}, \ldots, \delta_{i, s} \mathbf{W}_{l_{i, s}}\right],
$$

and

$$
f_{i, j}=\left[\delta_{i, 0} \mathbf{W}_{l_{i, 0}}, \ldots, \delta_{i, j-1} \mathbf{W}_{l_{i, j-1}}, \delta_{i, j+1} \mathbf{W}_{l_{i, j+1}}, \ldots, \delta_{i, s} \mathbf{W}_{l_{i, s}}\right],
$$

$j=0, \ldots, s$. Then, by (4.31) and (4.33), we have

$$
h_{i, j}=\beta_{i} s \frac{\operatorname{vol}\left(t_{i, j}\right)}{\operatorname{vol}\left(f_{i, j}\right)} .
$$


From (4.32) and (4.37) we can conclude that the choice (4.33), with

$$
\beta_{i}=\frac{H_{i}}{2 s} \max _{j=0, \ldots, s}\left\{\frac{\operatorname{vol}\left(f_{i, j}\right)}{\operatorname{vol}\left(t_{i, j}\right)}\right\},
$$

leads to an MPS-simplex satisfying the condition in Theorem 3.1. The following result is a direct consequence of (4.38).

Theorem 4.4. Given an interior vertex $\mathbf{V}_{i}$ of $\Delta^{s}$ and a set of vertices $\mathbf{V}_{l_{i, j}}, j=0, \ldots, s$, at the boundary of $\Omega_{i}$, let $\mathcal{D}_{i}$ be the set of any set $\delta:=\left\{\delta_{i, j}, j=0, \ldots, s\right\}$ with $\delta_{i, j} \in\{-1,1\}$ and such that the point $\mathbf{O}=(0, \ldots, 0)$ is contained in the s-simplex $\left[\delta_{i, 0} \mathbf{W}_{l_{i, 0}}, \ldots, \delta_{i, s} \mathbf{W}_{l_{i, s}}\right]$. The points $\mathbf{Q}_{i, j}, j=0, \ldots, s$, associated with vertex $\mathbf{V}_{i}$ can be chosen as in (4.17) such that the condition in Theorem 3.1 is satisfied and

$$
\left\|\mathbf{Q}_{i, j}-\mathbf{V}_{i}\right\|_{2}=\beta_{i, j}\left\|\mathbf{V}_{l_{i, j}}-\mathbf{V}_{i}\right\|_{2} \leq \frac{H_{i}}{2 s} \min _{\delta \in \mathcal{D}_{i}} \max _{k=0, \ldots, s}\left\{\frac{\operatorname{vol}\left(f_{i, k}\right)}{\operatorname{vol}\left(t_{i, k}\right)}\right\},
$$

with $t_{i, k}$ and $f_{i, k}$ given by (4.35)-(4.36).

Based on Theorem 4.4, we now propose an algorithm for the construction of an MPS-simplex for an interior vertex $\mathbf{V}_{i}$ such that the points $\mathbf{Q}_{i, j}$ belong to edges of $\Delta^{s}$ emanating from $\mathbf{V}_{i}$ or to their prolongation. The simplex has to contain the Bézier domain points in the direct neighbourhood of $\mathbf{V}_{i}$, and it must have a small (or preferably minimal) hyper-volume.

1. Determine (if it is possible) a set of $(s+1)$ vertices $\mathbf{V}_{l_{i, j}}, j=0, \ldots, s$, at the boundary of $\Omega_{i}$, and a set of values $\delta_{i, j} \in\{-1,1\}, j=0, \ldots, s$, such that

(a) the $s$-simplex $\left[\delta_{i, 0} \mathbf{W}_{l_{i, 0}}, \ldots, \delta_{i, s} \mathbf{W}_{l_{i, s}}\right]$ contains the point $(0, \ldots, 0)$ in its interior, with $\mathbf{W}_{l_{i, j}}$ defined by (4.34);

(b) it minimizes $\max _{k=0, \ldots, s}\left\{\frac{\operatorname{vol}\left(f_{i, k}\right)}{\operatorname{vol}\left(t_{i, k}\right)}\right\}$, with $t_{i, k}$ and $f_{i, k}$ defined by (4.35)-(4.36).

2. For each $j=0, \ldots, s$, select a point $\mathbf{Q}_{i, j}$ along the line through the points $\mathbf{V}_{i}$ and $\mathbf{V}_{l_{i, j}}$, such that the simplex $\left[\mathbf{Q}_{i, 0}, \ldots, \mathbf{Q}_{i, s}\right]$ has minimal hyper-volume and contains all Bézier domain points in the direct neighbourhood of $\mathbf{V}_{i}$, see Theorem 3.1.

This procedure is illustrated in Figure 6 for the case $s=2$. The left MPS-triangle is constructed using only $\beta_{i, j}>0$ in (4.17), so all $\delta_{i, j}=1$. In the construction of the right MPS-triangle also negative $\beta_{i, j}$ were allowed (thus, $\delta_{i, j} \in\{-1,1\}$ ).

The algorithm presented above must be suitably adapted in the case of boundary vertices. For such vertices, it may be preferred that $\mathbf{V}_{i}=\mathbf{Q}_{i, j}$, or that $\mathbf{V}_{i}$ is situated at an $m$-face $(m<s)$ spanned by some points $\mathbf{Q}_{i, j}$. For the sake of brevity we omit the related details.

\subsection{Numerical example}

We now illustrate numerically the presented quasi-interpolation method. Consider the well-known Franke bivariate test function [8],

$$
\begin{aligned}
f(x, y)= & \frac{3}{4} \exp \left(-\frac{(9 x-2)^{2}+(9 y-2)^{2}}{4}\right)+\frac{3}{4} \exp \left(-\frac{(9 x+1)^{2}}{49}-\frac{9 y+1}{10}\right) \\
& +\frac{1}{2} \exp \left(-\frac{(9 x-7)^{2}+(9 y-3)^{2}}{4}\right)-\frac{1}{5} \exp \left(-(9 x-4)^{2}-(9 y-7)^{2}\right),
\end{aligned}
$$



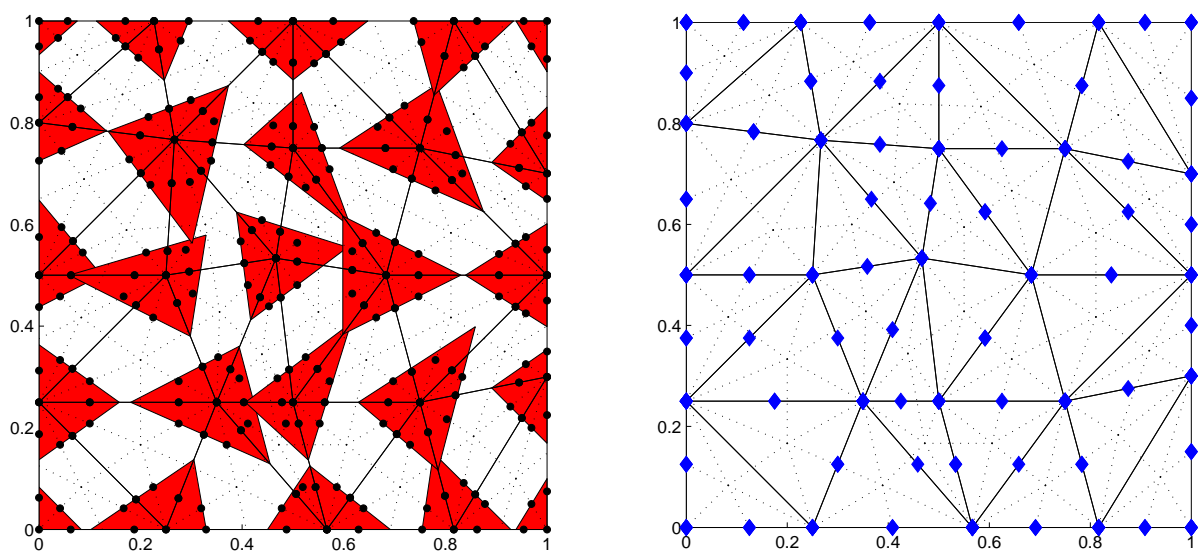

Figure 7: A triangulation with MPS-refinement. Left: MPS-triangles and MPS-points. Right: data sites used by the quasi-interpolant.

on the domain $\Omega=[0,1] \times[0,1]$. To measure the accuracy of a spline fit $s_{\mathrm{MPS}}$, we have computed the maximum error and the mean error on a $400 \times 400$ uniform grid $G$ on $\Omega$ :

$$
E_{\text {max }}=\max _{(x, y) \in G}\left|f(x, y)-s_{\mathrm{MPS}}(x, y)\right|, \quad E_{\text {mean }}=\frac{\sum_{(x, y) \in G}\left|f(x, y)-s_{\mathrm{MPS}}(x, y)\right|}{400 \times 400} .
$$

Figure 7 depicts the used triangulation $\Delta$ with a particular MPS-refinement. The MPS-triangles determining the set of B-splines are shown in the left picture. All the vertices of these triangles lie on edges of $\Delta$ or on their prolongations. For their construction we have followed the procedure described in Section 4.3. We have chosen a quasi-interpolant using only function values of $f$ at the vertices of $\Delta$ and at midpoints of edges of $\Delta$. So, we have taken the points $\mathbf{Z}_{i, j, k}$ as in (4.18) with $\rho_{i, j}=1 / 2$. These points are depicted in Figure 7 (right). The corresponding Powell-Sabin quasi-interpolant has a maximum error equal to 0.0665 , and its mean error is 0.0184 . The classical Powell-Sabin Hermite interpolant, defined by (4.1)-(4.2), has a maximum error of 0.1173 and a mean error of 0.0122 .

For other numerical examples that also fit into this multivariate quasi-interpolation framework, we refer to $[10,18,19]$.

\section{Conclusion}

In this paper, we have presented a method for constructing a normalized basis for the multivariate quadratic spline space defined over a generalized Powell-Sabin refinement of a triangulation in $\mathbb{R}^{s}$, $s \geq 1$. The basis functions have a local support, they are nonnegative, and they form a partition of unity. The construction has a geometric interpretation: it is equivalent to the determination of an $s$-simplex that must contain a specific set of points. We have shown that the univariate version is related to the well-known classical B-splines of degree two. Our construction is a generalization of the bivariate and trivariate basis described in [6] and [19].

We have also constructed a family of quasi-interpolants based on this B-spline representation. The quasi-interpolants are obtained via linear combinations of local function values. Given a suitable 
set of basis functions, the evaluation points can be chosen at the position of the vertices of the triangulation $\Delta^{s}$ and/or on edges of $\Delta^{s}$. This choice is very attractive in practical applications. We have provided an upper bound for the infinity norm of the quasi-interpolants, and in combination with the reproduction of quadratic polynomials, it guarantees the optimal approximation order of the quasi-interpolation schemes.

\section{Acknowledgement}

Hendrik Speleers is a Postdoctoral Fellow of the Research Foundation Flanders (Belgium).

\section{References}

[1] C. Bangert and H. Prautzsch. A geometric criterion for the convexity of Powell-Sabin interpolants and its multivariate generalization. Comput. Aided Geom. Design, 16:529-538, 1999.

[2] S.C. Brenner and L.R. Scott. The mathematical theory of finite element methods. SpringerVerlag, Second edition, 2002.

[3] C. Dagnino and P. Lamberti. On the construction of local quadratic spline quasi-interpolants on bounded rectangular domains. J. Comput. Appl. Math., 221:367-375, 2008.

[4] C. de Boor. B-form basics. In G. Farin, editor, Geometric Modeling: Algorithms and New Trends, pages 131-148. SIAM Publications, 1987.

[5] C. de Boor. A Practical Guide to Spline. Springer, Revised edition, 2001.

[6] P. Dierckx. On calculating normalized Powell-Sabin B-splines. Comput. Aided Geom. Design, 15:61-78, 1997.

[7] T. Dupont and R. Scott. Polynomial approximation of functions in Sobolev spaces. Math. Comp., 34:441-463, 1980 .

[8] R. Franke. Scattered data interpolation: tests of some methods. Math. Comp., 38:181-200, 1982.

[9] A.J. Hanson. Geometry for n-dimensional graphics. In P.S. Heckbert, editor, Graphics Gems IV, pages 149-170. Academic Press, San Diego, 1994.

[10] C. Manni and P. Sablonnière. Quadratic spline quasi-interpolants on Powell-Sabin partitions. Adv. Comput. Math., 26:283-304, 2007.

[11] G. Nürnberger, C. Rössl, H.P. Seidel, and F. Zeilfelder. Quasi-interpolation by quadratic piecewise polynomials in three variables. Comput. Aided Geom. Design, 22:221-249, 2005.

[12] M.J.D. Powell and M.A. Sabin. Piecewise quadratic approximations on triangles. ACM Trans. Math. Softw., 3:316-325, 1977.

[13] S. Remogna. Constructing good coefficient functionals for bivariate $C^{1}$ quadratic spline quasiinterpolants. In M. Dæhlen, M. Floater, T. Lyche, J.L. Merrien, K. Mørken, and L.L. Schumaker, editors, Mathematical Methods for Curves and Surfaces, LNCS 5862, pages 329-346. 2010 . 
[14] S. Remogna and P. Sablonnière. On trivariate blending sums of univariate and bivariate quadratic spline quasi-interpolants on bounded domains. Comput. Aided Geom. Design, 28:89101, 2011.

[15] M. Rhein and T. Kalbe. Quasi-interpolation by quadratic $C^{1}$-splines on truncated octahedral partitions. Comput. Aided Geom. Design, 26:825-841, 2009.

[16] P. Sablonnière. Error bounds for Hermite interpolation by quadratic splines on an $\alpha$ triangulation. IMA J. Numer. Anal., 7:495-508, 1987.

[17] P. Sablonnière. Recent progress on univariate and multivariate polynomial and spline quasiinterpolants. In M.G. de Bruijn, D.H. Mache, and J. Szabados, editors, Trends and Applications in Constructive Approximation, pages 229-245. Birkhäuser Verlag, 2005.

[18] D. Sbibih, A. Serghini, and A. Tijini. Polar forms and quadratic spline quasi-interpolants on Powell-Sabin partitions. Appl. Numer. Math., 59:938-958, 2009.

[19] D. Sbibih, A. Serghini, and A. Tijini. Normalized trivariate B-splines on Worsey-Piper split and quasi-interpolants. BIT Numer. Math., accepted, 2011.

[20] T. Sorokina and A.J. Worsey. A multivariate Powell-Sabin interpolant. Adv. Comput. Math., 29:71-89, 2008.

[21] H. Speleers. A normalized basis for quintic Powell-Sabin splines. Comput. Aided Geom. Design, 27:438-457, 2010.

[22] H. Speleers. A normalized basis for reduced Clough-Tocher splines. Comput. Aided Geom. Design, 27:700-712, 2010.

[23] H. Speleers. Construction of normalized B-splines for a family of smooth spline spaces over Powell-Sabin triangulations. Constr. Approx., accepted, 2011.

[24] A.J. Worsey and B. Piper. A trivariate Powell-Sabin interpolant. Comput. Aided Geom. Design, 5:177-186, 1988. 